\title{
Article \\ Network Pharmacology Study on Morus alba L. Leaves: Pivotal Functions of Bioactives on RAS Signaling Pathway and Its Associated Target Proteins against Gout
}

\author{
Ki Kwang Oh $\mathbb{B}$, Md. Adnan $\mathbb{1}$ and Dong Ha Cho* \\ Department of Bio-Health Convergence, College of Biomedical Science, Kangwon National University, \\ Chuncheon 24341, Korea; nivirna07@kangwon.ac.kr (K.K.O.); mdadnan1991.pharma@gmail.com (M.A.) \\ * Correspondence: chodh@kangwon.ac.kr; Tel.: +82-33-250-6475
}

Citation: Oh, K.K.; Adnan, M.; Cho, D.H. Network Pharmacology Study on Morus alba L. Leaves: Pivotal Functions of Bioactives on RAS Signaling Pathway and Its Associated Target Proteins against Gout. Int. J. Mol. Sci. 2021, 22, 9372. https:// doi.org/10.3390/ijms22179372

Academic Editor: Jessica Holien

Received: 30 July 2021

Accepted: 27 August 2021

Published: 29 August 2021

Publisher's Note: MDPI stays neutral with regard to jurisdictional claims in published maps and institutional affiliations.

Copyright: (c) 2021 by the authors. Licensee MDPI, Basel, Switzerland. This article is an open access article distributed under the terms and conditions of the Creative Commons Attribution (CC BY) license (https:// creativecommons.org/licenses/by/ $4.0 /)$.
Abstract: M. alba L. is a valuable nutraceutical plant rich in potential bioactive compounds with promising anti-gouty arthritis. Here, we have explored bioactives, signaling pathways, and key proteins underlying the anti-gout activity of $M$. alba L. leaves for the first-time utilizing network pharmacology. Bioactives in M. alba L. leaves were detected through GC-MS (Gas ChromatographyMass Spectrum) analysis and filtered by Lipinski's rule. Target proteins connected to the filtered compounds and gout were selected from public databases. The overlapping target proteins between bioactives-interacted target proteins and gout-targeted proteins were identified using a Venn diagram. Bioactives-Proteins interactive networking for gout was analyzed to identify potential ligand-target and visualized the rich factor on the R package via the Kyoto Encyclopedia of Genes and Genomes (KEGG) pathway on STRING. Finally, a molecular docking test (MDT) between bioactives and target proteins was analyzed via AutoDock Vina. Gene Set Enrichment Analysis (GSEA) demonstrated that mechanisms of $M$. alba L. leaves against gout were connected to 17 signaling pathways on 26 compounds. AKT1 (AKT Serine/Threonine Kinase 1), $\gamma$-Tocopherol, and RAS signaling pathway were selected as a hub target, a key bioactive, and a hub signaling pathway, respectively. Furthermore, three main compounds ( $\gamma$-Tocopherol, 4-Dehydroxy-N-(4,5-methylenedioxy-2-nitrobenzylidene) tyramine, and Lanosterol acetate) and three key target proteins-AKT1, PRKCA, and PLA2G2A associated with the RAS signaling pathway were noted for their highest affinity on MDT. The identified three key bioactives in M. alba L. leaves might contribute to recovering gouty condition by inactivating the RAS signaling pathway.

Keywords: Morus alba L. leaves; network pharmacology; gout; AKT1; $\gamma$-tocopherol; RAS signaling pathway

\section{Introduction}

Gout is a common and complex arthritis disease, often causing severe pain, swelling, redness and tenderness due to joint inflammation [1]. Gout is characterized by a disorder of uric acid crystal accumulation in blood, and its deposition is a vital factor to induce acute inflammation within and around joints [2]. Commonly, gouty flare-ups are unexpected and intense, more frequent at night [3]. In general, males are more likely than females to undergo symptoms of gout. More males are diagnosed between 30 and 50 years old, and females are more prevalent after menopause [4]. A report expounds that gout prevention is fundamental through lifestyle changes such as limiting alcohol, relieving stress, regular exercise, and taking enough herbal and dairy products [5]. Another report shows that Traditional Chinese Medicine (TCM) is used to treat gout with satisfactory effect [6]. Even though researchers are conducting experiments, there are no complete drugs for patients with gout. Existing drugs such as colchicines, corticosteroids, and non-steroidal anti-inflammatory drugs (NSAIDs) are utilized as an amelioration strategy against gout [7]. These medications might show good efficacy for a short time; however, for longer time 
periods, gastrointestinal, nausea, vomiting, and even renal toxicity could occur [8]. Therefore, herbal medicine might be a favorable remedy to diminish negative side effects during administration.

Morus alba L. is commonly distributed in Japan, India, China, and Korea, frequently used to alleviate joint pain, kidney and liver complication, and type 2 diabetes mellitus by tradition. Due to its rich nutritional value, $M$. alba L. leaves are cultivated as food for silkworms which produce high-quality silk [9]. Apart from silk production, M. alba L. leaves are of great biological and pharmacological interest to researchers. They contain diverse polyphenolic compounds with potent antioxidant, anticancer, and anti-inflammatory effects [10-12]. Recent research revealed that methanolic extract of M. alba L. leaves notably diminished neutropenia, elevated phagocytic index, and evidently fostered immunomodulatory effects [13]. An animal experiment exposed that administration of $M$. alba L. leaves (70\% methanolic extract) significantly reduced uric acid level in plasma and showed potent antioxidant activity in mice [14]. Another study concluded that M. alba L. leaves have potent anti-inflammatory and antioxidant activities that might be an excellent candidate to relieve gouty arthritis pain [15]. Moreover, $M$. alba L. leaves ethanolic extract is a potent inhibitor of xanthine oxidase $(\mathrm{XO})$ enzyme associated directly with hyperuricemia [16]. Although many researchers proved to have promising analgesic, anti-inflammatory, and anti-arthritis potentials of M. alba L. leaves [17-19], however, the key bioactive compounds and mechanisms of $M$. alba L. leaves against gouty arthritis have not been established completely. M. alba L. leaves shed light on medicinal effects to alleviate symptoms of gout as well as a potent antagonist of $\mathrm{XO}$.

Hence, our goal is to substantiate bioactives and mechanisms of Morus alba (M. alba) L. leaves against gout as Morus alba (M. alba) L. leaves have been reported as an important herbal medicine to counteract gout. Our study used GC-MS analysis with ChemStation integrated algorithms to maximize the discovery of drug-likeness bioactives in M. alba L. leaves.

System biology has been focused on the multiple interactions in biology research from a whole viewpoint instead of adjusting to a single molecule [20]. For example, network pharmacology is utilized to identify multiple factors to interpret therapeutic compounds, toxicants, signaling pathways, hub proteins, and mechanisms of phytochemicals against various diseases [21,22]. With a systemic approach, network pharmacology can decode novel mechanism(s) of action which mainly focus on "multiple targets, multiple drugs" rather than "one target, one drug" [23,24]. The network pharmacology is a useful tool for constructing a compound-target-signaling pathway network through the overall perspective, and this holistic approach is very efficient for evaluating bioactive compounds $[25,26]$. However, in this study, network pharmacology was implemented to explore the bioactive constituents and mechanisms of M. alba L. leaves against gout. The brief analysis step of this study is displayed in Figure 1. 


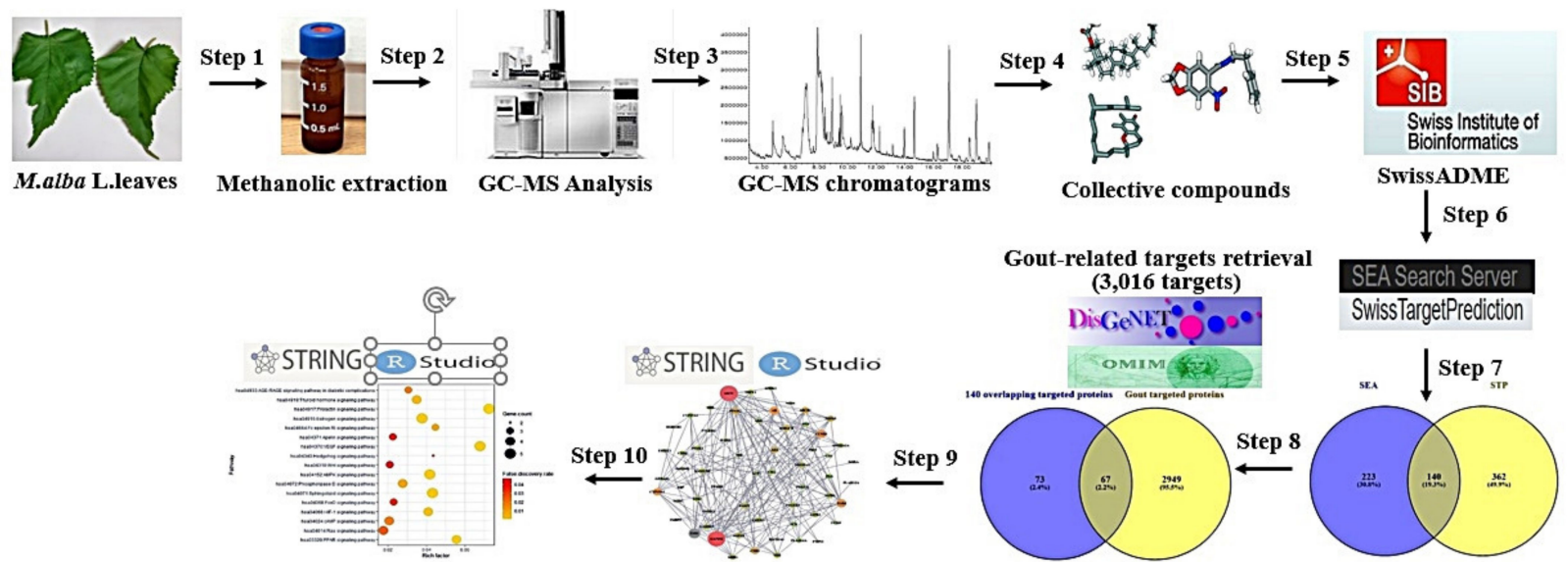

17 signaling pathways of M.alba L. leaves against Gout PPI networks 140 overlapping target proteins identification 725 target proteins identification Step 11

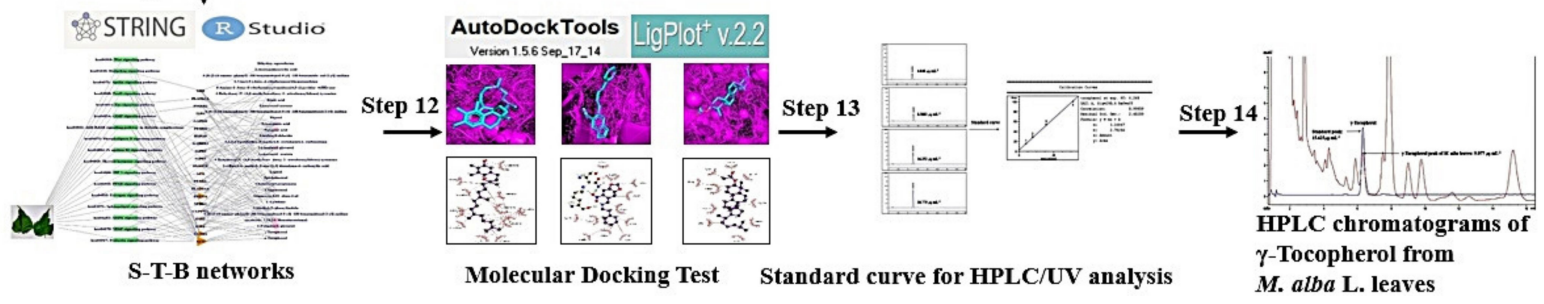

Figure 1. Workflow of network pharmacology analysis of M. alba L. leaves against Gout.

\section{Results}

\subsection{Physicochemical Properties of Potential Chemical Compositions from M. alba L. Leaves}

A total of 36 bioactives in $M$. alba L. leaves were identified via GC-MS analysis (Figure 2), and the name of compounds, retention time, peak area (\%), Pubchem ID was presented in Table 1. All 36 bioactives were satisfied by Lipinski's rule (Molecular Weight $\leq 500 \mathrm{~g} / \mathrm{mol}$; Moriguchi octanol-water partition coefficient $\leq 4.15$; Number of Nitrogen or Oxygen $\leq 10$; Number of $\mathrm{NH}$ or $\mathrm{OH} \leq 5$ ). The TPSA value of all bioactives was also accepted (Table 2).

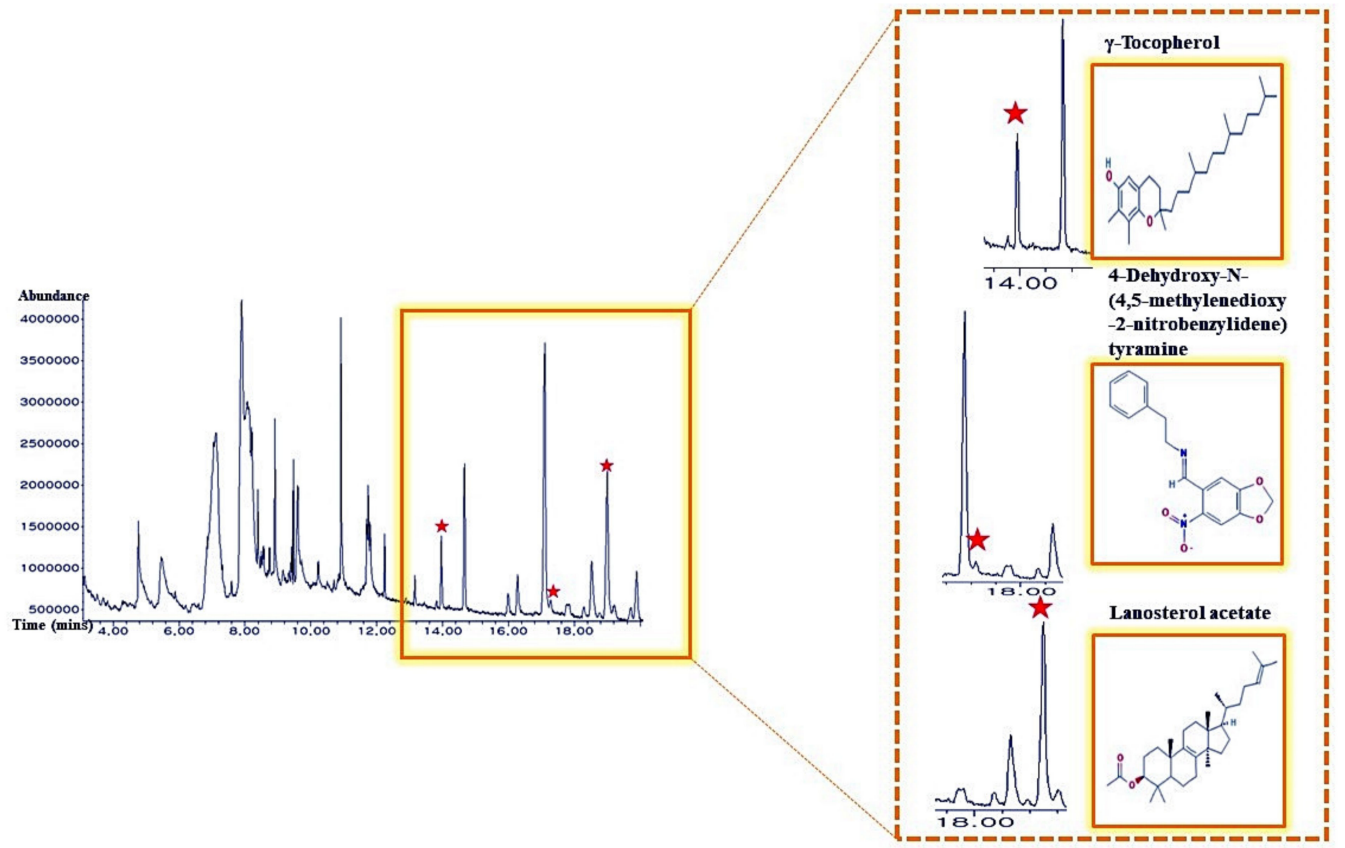

Figure 2. A typical GC-MS chromatogram of methanolic extract of M. alba L. leaves and indication of 3 main bioactives. 
Table 1. A list of the identified 36 chemical compounds from M. alba L. leaves through GC-MS.

\begin{tabular}{|c|c|c|c|c|c|}
\hline No. & Compound Name & Retention Time (min) & Area $(\%)$ & Pubchem ID & $\begin{array}{c}\text { Pharmacological } \\
\text { Activities (Reference) }\end{array}$ \\
\hline 1 & Propanal, 2,3-dihydroxy- & 3.702 & 0.16 & 751 & No activities [27] \\
\hline 2 & 4-Oxopentyl formate & 4.298 & 0.45 & 536673 & No activities [27] \\
\hline 3 & Piperazine, 2,5-dimethyl-, cis- & 4.52 & 0.34 & 7816 & No activities [27] \\
\hline 4 & $\begin{array}{l}\text { 2,3-Dihydro-3,5-dihydroxy-6-methyl-4h- } \\
\text { pyran-4-one }\end{array}$ & 4.779 & 3.14 & 119838 & Antioxidant [28] \\
\hline 5 & $\begin{array}{l}\text { 2-Vinyl-9-[.beta.-d- } \\
\text { ribofuranosyl]hypoxanthine }\end{array}$ & 5.164 & 0.25 & 135493011 & No activities [27] \\
\hline 6 & Thiocyanic acid, 2-propynyl ester & 5.471 & 3.94 & 123411 & Anticytotoxicity [29] \\
\hline 7 & 2-Acetamidoacrylic acid & 6.471 & 0.47 & 79482 & No activities [27] \\
\hline 8 & L-Cytidine & 7.125 & 16.32 & 122948 & No activities [27] \\
\hline 9 & $\begin{array}{l}\text { 6-Amino-1-.beta.-d-ribofuranosylimidazo } \\
\text { [4,5-c]pyridin-4(5H)-one }\end{array}$ & 7.596 & 0.7 & 545638 & No activities [27] \\
\hline 10 & Kinic acid & 7.904 & 12.09 & 1064 & Cox-2 inhibitor [27] \\
\hline 11 & $\begin{array}{l}\text { 2-t-Butyl-4-methyl-5-oxo-[1,3]dioxolane- } \\
\text { 4-carboxylic acid }\end{array}$ & 8.077 & 14.34 & 545703 & No activities [27] \\
\hline 12 & 1-(4-Bromobutyl)-2-piperidinone & $8.404,8.558,8.750$ & 5.15 & 536377 & No activities [27] \\
\hline 13 & Palmitic acid & $8.914,9.164$ & 4.71 & 985 & Antibacterial [27] \\
\hline 14 & Phytol & 9.471 & 2.49 & 145386 & Antitumor [30] \\
\hline 15 & Linoleoyl chloride & 9.606 & 4.76 & 9817754 & Anti-arteriosclerosis [31] \\
\hline 16 & 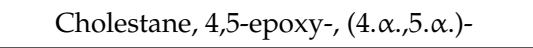 & 10.231 & 2.39 & 537014 & No activities [27] \\
\hline 17 & Tricosanoic acid & 10.5 & 0.7 & 17085 & No activities [27] \\
\hline 18 & $\begin{array}{l}\text { 1,2,3,4-Tetrahydro-9-methyl-6-cyclohexyl- } \\
\text { 1-carbazolone }\end{array}$ & 10.712 & 0.36 & 535444 & No activities [27] \\
\hline 19 & 1-Palmitoylglycerol & 10.923 & 3.57 & 14900 & No activities [27] \\
\hline 20 & 2-Linoleoylglycerol (beta-Monolinolein) & 11.702 & 1.14 & 5365676 & Anti-breast cancer [32] \\
\hline 21 & cis,cis,cis-7,10,13-Hexadecatrienal & 11.741 & 1.27 & 5367366 & Antibacterial [33] \\
\hline 22 & Cholesteryl propionate & 11.808 & 1.44 & 313255 & No activities [27] \\
\hline 23 & $\begin{array}{l}\text { Curan-17-oic acid, } \\
\text { 2,16-didehydro-20-hydroxy-19-oxo-, } \\
\text { methyl ester }\end{array}$ & 12.241 & 0.43 & 550468 & Anti-yeast [34] \\
\hline 24 & $\begin{array}{l}\text { 4-[6-[2-(4-aminophenyl)-3H- } \\
\text { benzimidazol-5-yl]-1H-benzimidazol-2- } \\
\text { yl]aniline }\end{array}$ & 13.818 & 0.12 & 1365597 & No activities [27] \\
\hline 25 & $\gamma$-Tocopherol & 13.962 & 0.85 & 14986 & Antioxidant [35] \\
\hline 26 & $\alpha$-Tocopherol & 14.664 & 1.95 & 14985 & Antioxidant [35] \\
\hline 27 & 4-Hydroxywarfarin & 15.991 & 0.52 & 54682146 & No activities [27] \\
\hline 28 & Stigmasta-5,22-dien-3-ol & 16.279 & 0.82 & 6432745 & Antiviral [36] \\
\hline 29 & Clionasterol & 17.096 & 5.48 & 457801 & Anticomplementary [37] \\
\hline 30 & Epicholestrol & 17.779 & 0.36 & 304 & No activities [27] \\
\hline 31 & $\begin{array}{l}\text { 4-Dehydroxy-N-(4,5-methylenedioxy-2- } \\
\text { nitrobenzylidene)tyramine }\end{array}$ & 17.846 & 0.25 & 610062 & Antibacterial [38] \\
\hline 32 & Lupeol & 18.529 & 1.58 & 259846 & Anticancer, Antiviral [27] \\
\hline 33 & Lanosterol acetate & 19 & 3.4 & 3036237 & No activities [27] \\
\hline 34 & Dihydroagarofuran & 19.212 & 0.43 & 21593552 & Neuroprotective [39] \\
\hline 35 & 2-Methyl-7-phenylindole & 19.721 & 0.3 & 610181 & Antibacterial [40] \\
\hline 36 & Lupenyl acetate & 19.894 & 1.11 & 6432150 & Skin cell proliferation [41] \\
\hline
\end{tabular}


Table 2. Physicochemical properties of bioactives for good oral bioavailability and cell membrane permeability.

\begin{tabular}{|c|c|c|c|c|c|c|c|c|}
\hline \multirow{3}{*}{ No. } & \multirow{3}{*}{ Compounds } & \multicolumn{4}{|c|}{ Lipinski Rules } & \multirow{2}{*}{ Lipinski's Violations } & \multirow{2}{*}{ Biavailability Score } & \multirow{2}{*}{$\operatorname{TPSA}\left(\AA^{2}\right)$} \\
\hline & & MW & HBA & HBD & MLog P & & & \\
\hline & & $<500$ & $<10$ & $\leq \mathbf{5}$ & $\leq 4.15$ & $\leq 1$ & $>0.1$ & $<140$ \\
\hline 1 & Propanal, 2,3-dihydroxy- & 90.08 & 3 & 2 & -1.66 & 0 & 0.55 & 57.53 \\
\hline 2 & 4-Oxopentyl formate & 130.14 & 3 & 0 & 0.28 & 0 & 0.55 & 43.37 \\
\hline 3 & Piperazine, 2,5-dimethyl-, cis- & 114.19 & 2 & 2 & 0.21 & 0 & 0.55 & 24.06 \\
\hline 4 & 2,3-Dihydro-3,5-dihydroxy-6-methyl-4h-pyran-4-one & 144.13 & 4 & 2 & -1.77 & 0 & 0.85 & 66.76 \\
\hline 5 & 2-Vinyl-9-[.beta.-d-ribofuranosyl]hypoxanthine & 294.26 & 7 & 4 & -1.77 & 0 & 0.55 & 133.49 \\
\hline 6 & Thiocyanic acid, 2-propynyl ester & 97.14 & 1 & 0 & 1.98 & 0 & 0.55 & 44.45 \\
\hline 7 & 2-Acetamidoacrylic acid & 129.11 & 3 & 2 & -0.63 & 0 & 0.85 & 66.40 \\
\hline 8 & L-Cytidine & 243.22 & 6 & 4 & -2.29 & 0 & 0.55 & 130.83 \\
\hline 9 & $\begin{array}{l}\text { 6-Amino-1-.beta.-d-ribofuranosylimidazo[4,5-c]pyridin- } \\
\text { 4(5H)-one }\end{array}$ & 282.25 & 6 & 5 & -2.51 & 0 & 0.55 & 146.62 \\
\hline 10 & Kinic acid & 192.17 & 6 & 5 & -2.14 & 0 & 0.56 & 118.22 \\
\hline 11 & 2-t-Butyl-4-methyl-5-oxo-[1,3]dioxolane-4-carboxylic acid & 2012.2 & 5 & 1 & 0.43 & 0 & 0.85 & 72.83 \\
\hline 13 & Palmitic acid & 256.42 & 2 & 1 & 4.19 & 1 & 0.85 & 37.30 \\
\hline 14 & Phytol & 296.53 & 1 & 1 & 5.25 & 1 & 0.55 & 20.23 \\
\hline 15 & Linoleoyl chloride & 298.89 & 1 & 0 & 4.82 & 1 & 0.55 & 17.07 \\
\hline 16 & 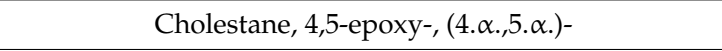 & 386.65 & 1 & 0 & 6.48 & 1 & 0.55 & 12.53 \\
\hline 17 & Tricosanoic acid & 354.61 & 2 & 1 & 5.79 & 1 & 0.85 & 37.30 \\
\hline 18 & 1,2,3,4-Tetrahydro-9-methyl-6-cyclohexyl-1-carbazolone & 281.39 & 1 & 1 & 3.51 & 0 & 0.55 & 22.00 \\
\hline 19 & 1-Palmitoylglycerol & 330.5 & 4 & 2 & 3.18 & 0 & 0.55 & 66.76 \\
\hline 20 & 2-Linoleoylglycerol (beta-Monolinolein) & 354.52 & 4 & 2 & 3.42 & 0 & 0.55 & 66.76 \\
\hline 21 & cis,cis,cis-7,10,13-Hexadecatrienal & 234.38 & 1 & 0 & 4.01 & 0 & 0.55 & 17.07 \\
\hline 22 & Cholesteryl propionate & 442.72 & 2 & 0 & 6.7 & 1 & 0.55 & 26.30 \\
\hline 23 & $\begin{array}{l}\text { Curan-17-oic acid, 2,16-didehydro-20-hydroxy-19-oxo-, } \\
\text { methyl ester }\end{array}$ & 354.4 & 5 & 2 & 1.17 & 0 & 0.55 & 78.87 \\
\hline 24 & $\begin{array}{l}\text { 4-[6-[2-(4-aminophenyl)-3H-benzimidazol-5-yl]-1H- } \\
\text { benzimidazol-2-yl]aniline }\end{array}$ & 416.48 & 2 & 4 & 3.34 & 0 & 0.55 & 109.40 \\
\hline
\end{tabular}


Table 2. Cont.

\begin{tabular}{|c|c|c|c|c|c|c|c|c|}
\hline \multirow{3}{*}{ No. } & \multirow{3}{*}{ Compounds } & \multicolumn{4}{|c|}{ Lipinski Rules } & \multirow{2}{*}{ Lipinski's Violations } & \multirow{2}{*}{ Biavailability Score } & \multirow{2}{*}{$\operatorname{TPSA}\left(\AA^{2}\right)$} \\
\hline & & MW & HBA & HBD & MLog P & & & \\
\hline & & $<500$ & $<10$ & $\leq 5$ & $\leq 4.15$ & $\leq 1$ & $>0.1$ & $<140$ \\
\hline 25 & $\gamma$-Tocopherol & 416.68 & 2 & 1 & 5.94 & 1 & 0.55 & 29.46 \\
\hline 26 & $\alpha$-Tocopherol & 430.71 & 2 & 1 & 6.14 & 1 & 0.55 & 29.46 \\
\hline 27 & 4-Hydroxywarfarin & 324.33 & 5 & 2 & 1.95 & 0 & 0.55 & 87.74 \\
\hline 28 & Stigmasta-5,22-dien-3-ol & 412.69 & 1 & 1 & 6.62 & 1 & 0.55 & 20.23 \\
\hline 29 & Clionasterol & 414.71 & 1 & 1 & 6.73 & 1 & 0.55 & 20.23 \\
\hline 30 & Epicholestrol & 386.65 & 1 & 1 & 6.34 & 1 & 0.55 & 20.23 \\
\hline 31 & $\begin{array}{l}\text { 4-Dehydroxy-N-(4,5-methylenedioxy-2- } \\
\text { nitrobenzylidene)tyramine }\end{array}$ & 298.29 & 5 & 0 & 1.49 & 0 & 0.55 & 76.64 \\
\hline 32 & Lupeol & 426.72 & 1 & 1 & 6.92 & 1 & 0.55 & 20.23 \\
\hline 33 & Lanosterol acetate & 468.75 & 2 & 0 & 6.98 & 1 & 0.55 & 26.30 \\
\hline 34 & Dihydroagarofuran & 222.37 & 1 & 0 & 3.81 & 0 & 0.55 & 9.23 \\
\hline 35 & 2-Methyl-7-phenylindole & 207.27 & 0 & 1 & 3.32 & 0 & 0.55 & 15.79 \\
\hline
\end{tabular}

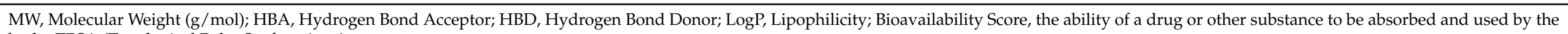
body; TPSA (Topological Polar Surface Area). 


\subsection{Overlapping Target Proteins between SEA and STP Associated with 36 Compounds}

A total of 363 target proteins from SEA and 502 target proteins from STP interacted with 36 compounds were extracted through SMILES format (Supplementary Table S1). Venn diagram showed that 140 target proteins were overlapping between the two public databases (Figure 3A).

SEA

STP

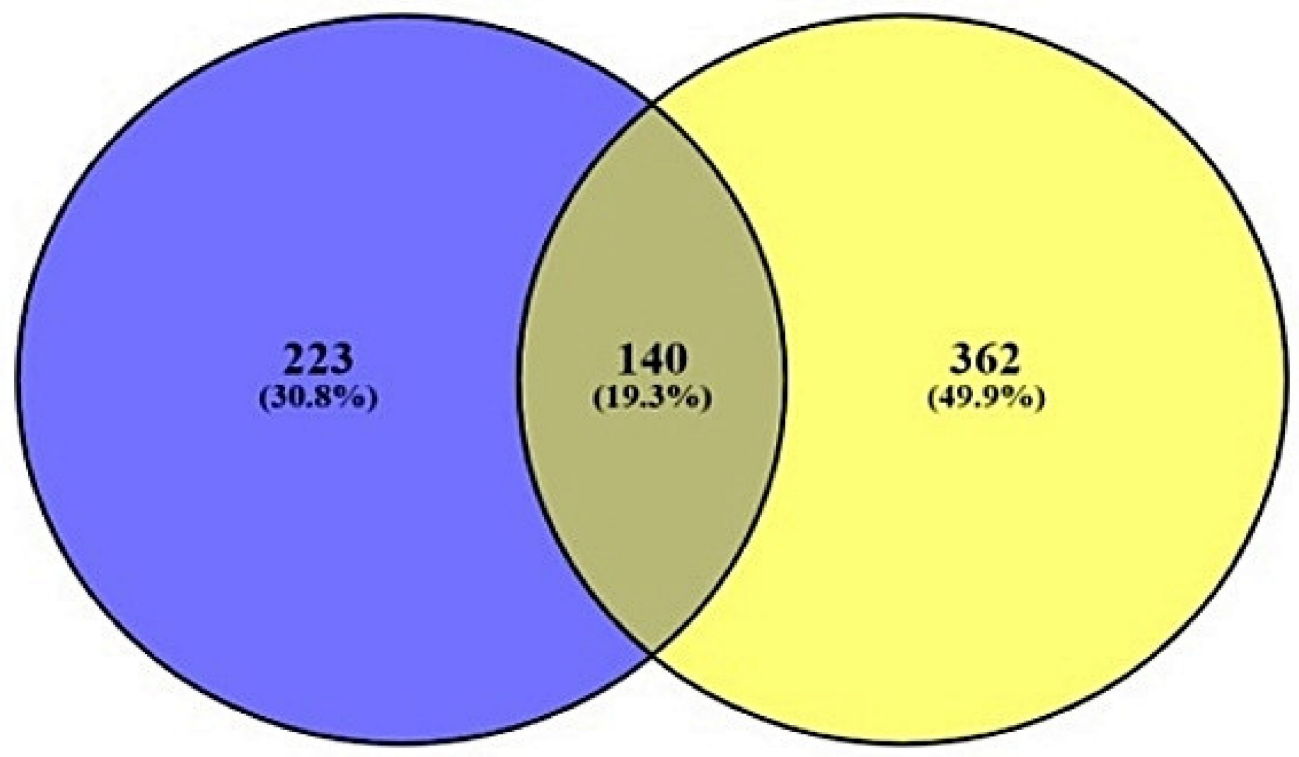

(A)

\section{0 overlapping targeted proteins Gout targeted proteins}

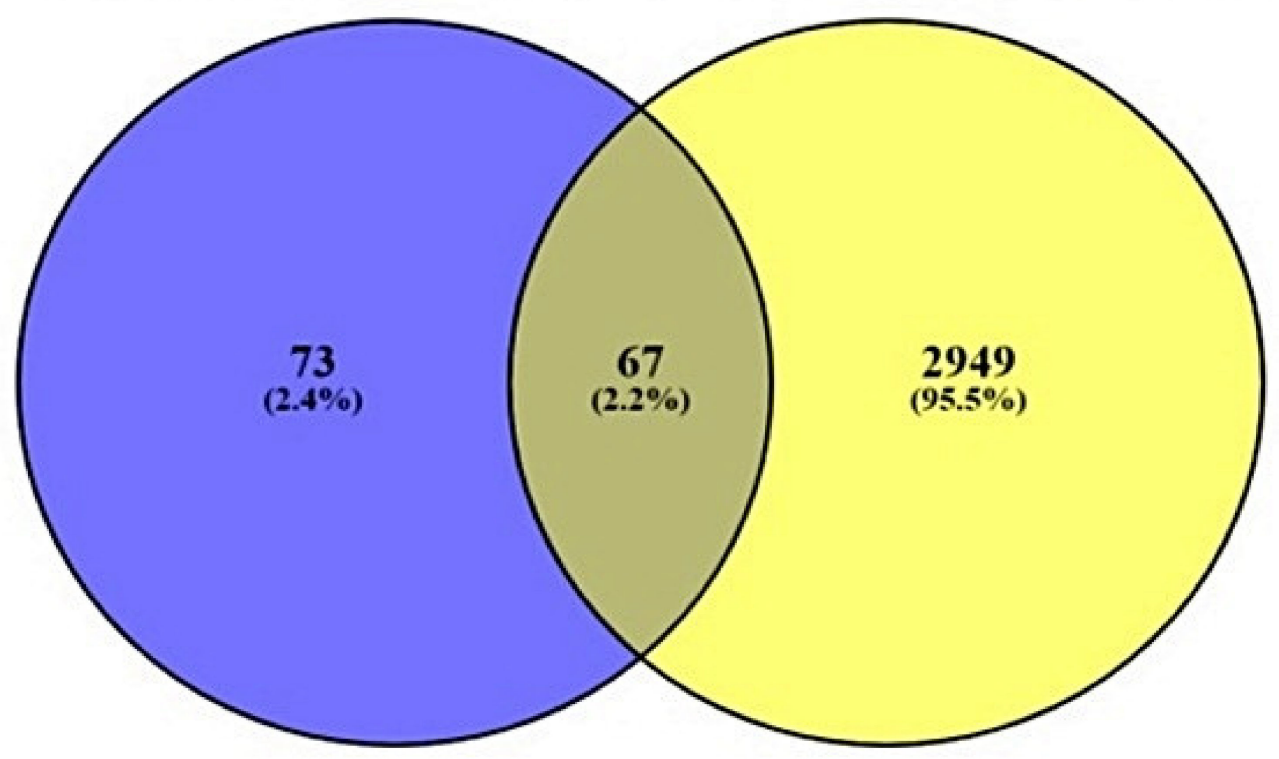

(B)

Figure 3. (A) Overlapping target proteins (140 target proteins) between SEA (223 target proteins) and STP (362 target proteins) (B) Overlapping target proteins between 140 overlapping target proteins from two databases (SEA and STP) and gout associated with target proteins (3016 target proteins). 
2.3. Overlapping Target Proteins between Gout-Related Target Proteins and the 140 Overlapping Target Proteins

A total of 3016 target proteins connected to gout were selected by retrieving DisGeNET and OMIM databases (Supplementary Table S2). Venn diagram displayed that 67 overlapping target proteins were identified between the 3016 target proteins and the 140 overlapping target proteins (Figure 3B) and (Supplementary Table S2).

\subsection{Protein-Protein Interaction from 60 Overlapping Target Proteins}

From STRING analysis, 60 out of 67 overlapping target proteins were closely interacted with each other, indicating 60 nodes and 199 edges (Figure 4). The removed 7 target proteins (HPSE, PAM, CA1, GSTK1, SLC5A2, GRK1, and BCHE) did not correlate within the overlapping 67 target proteins. In protein-protein interaction (PPI), the AKT1 target exhibited the highest degree (31) and is considered as a hub target protein (Table 3).

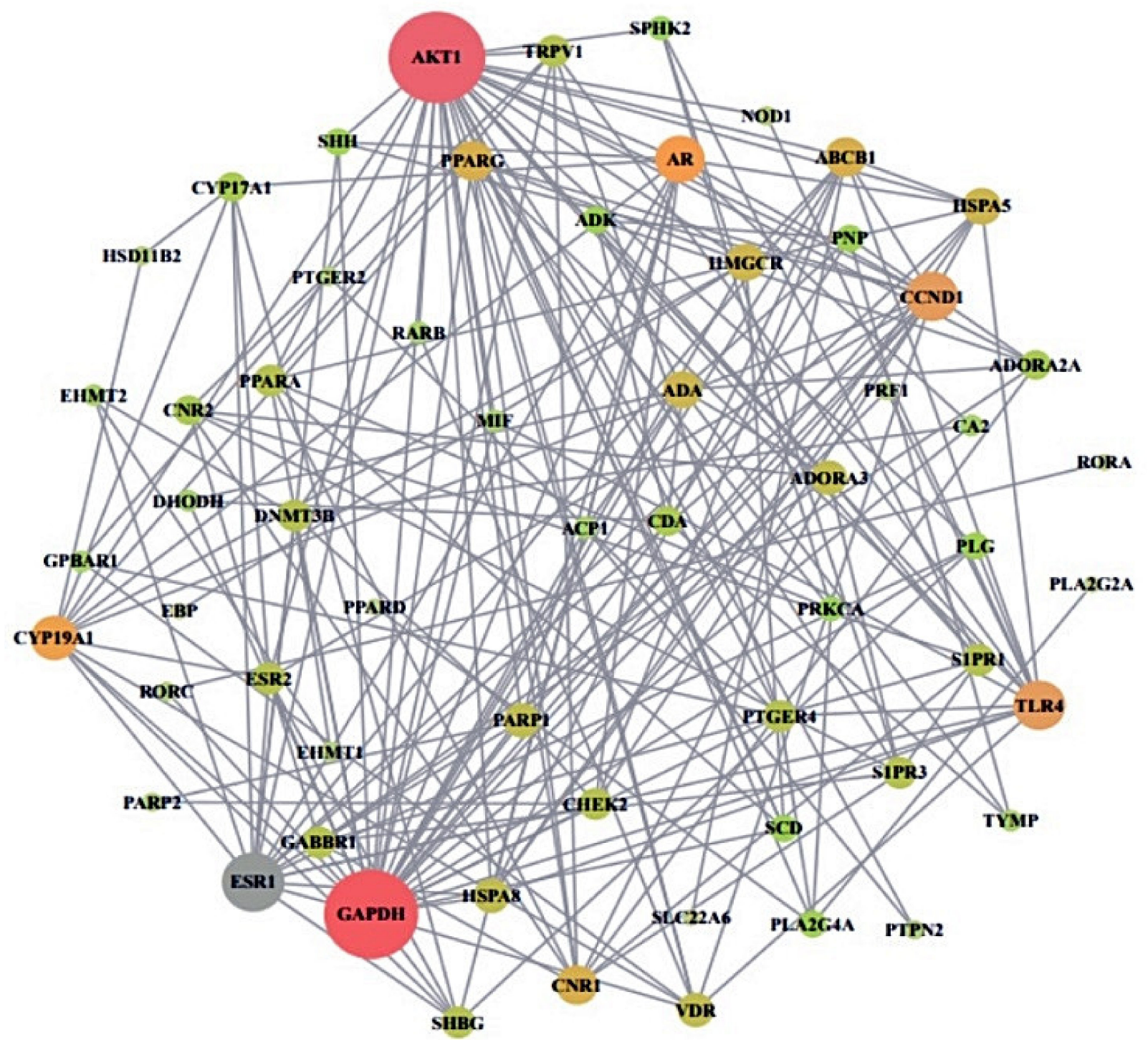

Figure 4. PPI networks of final overlapping 60 target proteins (60 nodes and 199 edges). Nodes: The number of networking proteins; Edges: Interactions between protein(s) and protein(s). 
Table 3. The degree value of 60 target proteins.

\begin{tabular}{cccccc}
\hline No. & Gene Symbol & Degree & No. & Gene Symbol & Degree \\
\hline 1 & AKT1 & 31 & 31 & CYP17A1 & 6 \\
2 & GAPDH & 30 & 32 & ADK & 5 \\
3 & ESR1 & 18 & 33 & PLA2G4A & 5 \\
4 & CCND1 & 14 & 34 & PLG & 5 \\
5 & TLR4 & 14 & 35 & PNP & 5 \\
6 & AR & 13 & 36 & SHH & 5 \\
7 & CYP19A1 & 12 & 37 & SCD & 5 \\
8 & ABCB1 & 10 & 38 & PRKCA & 4 \\
9 & CNR1 & 10 & 39 & SPHK2 & 4 \\
10 & PPARG & 10 & 40 & ACP1 & 3 \\
11 & ADA & 9 & 41 & CA2 & 3 \\
12 & HMGCR & 9 & 42 & DHODH & 3 \\
13 & HSPA5 & 9 & 43 & EHMT2 & 3 \\
14 & ADORA3 & 8 & 44 & GPBAR1 & 3 \\
15 & HSPA8 & 8 & 45 & MIF & 3 \\
16 & PARP1 & 8 & 46 & RARB & 3 \\
17 & VDR & 8 & 47 & TYMP & 3 \\
18 & CHEK & 7 & 48 & EHMT1 & 2 \\
19 & DNMT3B & 7 & 49 & NOD1 & 2 \\
20 & TRPV1 & 7 & 50 & PRF1 & 2 \\
21 & ESR2 & 7 & 51 & PTGER2 & 2 \\
22 & GABBR1 & 7 & 52 & PTPN2 & 2 \\
23 & PPARA & 7 & 53 & PARP2 & 2 \\
24 & PTGER4 & 7 & 54 & HSD11B2 & 2 \\
25 & S1PR1 & 7 & 55 & RORC & 2 \\
26 & S1PR3 & 7 & 56 & EBP & 1 \\
27 & SHBG & 7 & 57 & PLA2G2A & 1 \\
28 & ADORA2A & 6 & 58 & RORA & 1 \\
29 & CDA & 6 & 59 & PPARD & 1 \\
30 & CNR2 & 6 & 60 & & 1 \\
\hline & & & &
\end{tabular}

2.5. The 17 Signaling Pathways and Finding of a Hub Signaling of M. alba L. Leaves against Gout

The KEGG pathway enrichment analysis demonstrated that 67 target proteins were associated with 17 signaling pathways (False Discovery Rate $<0.05$ ). The 17 signaling pathways were directly related to gout development, exhibiting that these pathways might be the significant signal transduction of $M$. alba L. leaves against gout. The description of 17 signaling pathways was presented in Table 4. Additionally, a bubble plot suggested that the RAS (Renin Angiotensin System) signaling pathway might be a hub signaling pathway of M. alba L. leaves against gout (Figure 5).

Table 4. Target proteins in 17 signaling pathways enrichment related to gout.

\begin{tabular}{|c|c|c|}
\hline KEGG ID \& Description & Target Proteins & False Discovery Rate \\
\hline hsa04917:Prolactin signaling pathway & AKT1,CCND1,ESR1,ESR2,CYP17A1 & 0.0004 \\
\hline hsa04370:VEGF signaling pathway & AKT1,SPHK2,PRKCA,PLA2G4A & 0.0019 \\
\hline hsa04152:AMPK signaling pathway & AKT1,CCND1,PPARG,SCD,HMGCR & 0.0019 \\
\hline hsa04071:Sphingolipid signaling pathway & AKT1,S1PR1,S1PR3,SPHK2,PRKCA & 0.0019 \\
\hline hsa04915:Estrogen signaling pathway & AKT1,GABBR1,ESR1,ESR2,HSPA8 & 0.0022 \\
\hline hsa03320:PPAR signaling pathway & PPARA,PPARG,PPAR,SCD & 0.0024 \\
\hline hsa04066:HIF-1 signaling pathway & AKT1,GAPDH,TLR4,PRKCA & 0.0045 \\
\hline hsa04919:Thyroid hormone signaling pathway & AKT1,CCND1,ESR1 & 0.0069 \\
\hline hsa04664:Fc epsilon RI signaling pathway & AKT1,PRKCA,PLA2G4A & 0.012 \\
\hline hsa04072:Phospholipase D signaling pathway & AKT1,SPHK2,PRKCA,PLA2G4A & 0.012 \\
\hline $\begin{array}{l}\text { hsa04933:AGE-RAGE signaling pathway in } \\
\text { diabetic complications }\end{array}$ & AKT1,CCND1,PRKCA & 0.0232 \\
\hline hsa04024:cAMP signaling pathway & AKT1,PPARA,GABBR1 & 0.0232 \\
\hline hsa04014:Ras signaling pathway & AKT1,PRKCA,PLA2G2A,PLA2G4A & 0.0318 \\
\hline hsa04068:FoxO signaling pathway & AKT1,CCND1,S1PR1 & 0.0391 \\
\hline hsa04371:Apelin signaling pathway & AKT1,CCND1,SPHK2 & 0.0408 \\
\hline hsa04340:Hedgehog signaling pathway & SHH,CCND1 & 0.0416 \\
\hline hsa04310:Wnt signaling pathway & CCND1,PRKCA,PPARA & 0.0468 \\
\hline
\end{tabular}




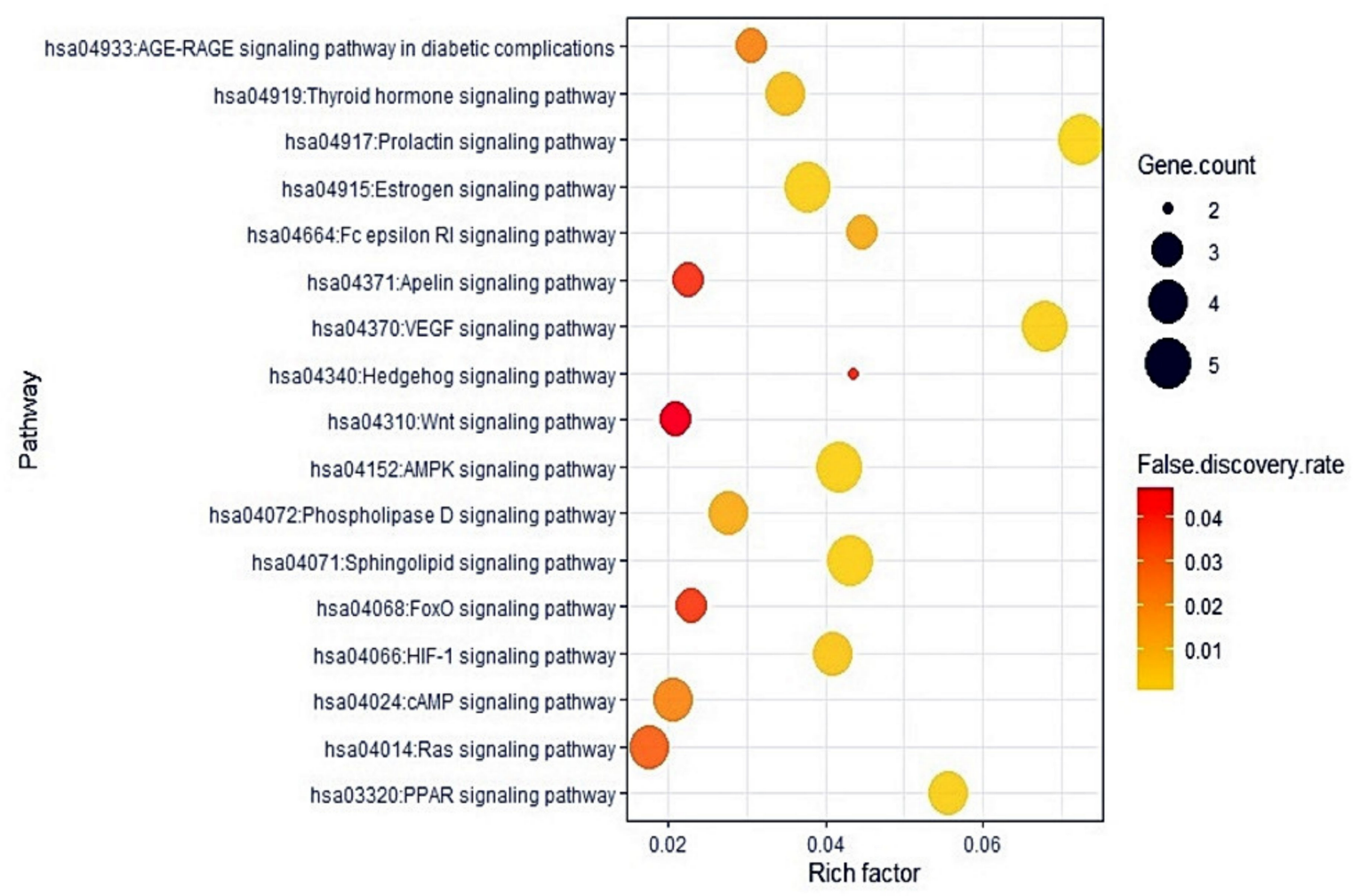

Figure 5. Bubble chart of 17 signaling pathways linked to the occurrence and progression of gout.

\subsection{A Signaling Pathway-Target Protein-Bioactive Networks}

A signaling pathway- target protein- bioactive (S-T-B) networks of M. alba L. leaves were displayed in Figure 6. There were 26 bioactives, 21 target proteins, and 17 pathways (64 nodes, 177 edges). The nodes represent a total number of bioactives, target proteins, and pathways. The edges indicate relationships of the three components. The S-T-B networks suggest that the network might interact with therapeutic efficacy against gout. The AKT1 is the most significant target with the highest degree value (14) among 17 signaling pathways related to 21 target proteins linked directly to the RAS signaling pathway.

\subsection{MDT Results of 4 Target Proteins and 4 Compounds Related to RAS Signaling Pathway}

Through the analysis of SEA and STP database, it was revealed that AKT1 was linked to four compounds ( $\gamma$-Tocopherol, $\alpha$-Tocopherol, 1-Palmitoylglycerol, and cis, cis, cis-7, 10, 13-Hexadecatrienal), PRKCA was associated with seven compounds (1-Palmitoylglycerol, 2Linoleoylglycerol, Linoleoyl chloride, Palmitic acid, Tricosanoic acid, Phytol, and 4-Dehydroxy$\mathrm{N}-(4,5$-methylenedioxy-2-nitrobenzylidene) tyramine), PLA2G2A was linked to 5 compounds (1-Palmitoylglycerol, Linoleoyl chloride, Palmitic acid, Tricosanoic acid, and Lanosterol acetate), PLA2G4A was linked to 5 compounds (2-Linoleoylglycerol, Linoleoyl chloride, Palmitic acid, Tricosanoic acid, and cis, cis, cis-7, 10, 13-Hexadecatrienal. The MDT was performed to evaluate these four proteins' binding energy against each related gene, individually. The docking figures were depicted in Figure 7A-C. The MDT score of four ligands on AKT1 protein (PDB ID: 4GV1) was analyzed in the "Homo Sapiens" mode. It was observed that $\gamma$-Tocopherol $(-7.3 \mathrm{kcal} / \mathrm{mol})$ docked on AKT1 exposed the most excellent binding energy, followed by $\alpha$-Tocopherol $(-7.0 \mathrm{kcal} / \mathrm{mol})$, 1-Palmitoylglycerol $(-6.9 \mathrm{kcal} / \mathrm{mol})$, and cis-ciscis-7,10,13-Hexadecatrienal $(-4.8 \mathrm{kcal} / \mathrm{mol})$. The detailed information was enlisted in Table 5. The MDT score of seven ligands on PRKCA protein (PDB ID: 3IW4) was conducted in the "Homo Sapiens" mode. It was exposed that 4-Dehydroxy-N-(4,5-methylenedioxy-2nitrobenzylidene)tyramine $(-8.4 \mathrm{kcal} / \mathrm{mol})$ docked on PRKCA manifested the most significant binding energy, followed by 2-Linoleoylglycerol $(-6.9 \mathrm{kcal} / \mathrm{mol})$, 1-Palmitoylglycerol 
$(-6.6 \mathrm{kcal} / \mathrm{mol})$, Tricosanoic acid $(-6.5 \mathrm{kcal} / \mathrm{mol})$, Phytol $(-5.6 \mathrm{kcal} / \mathrm{mol})$, Palmitic acid $(-5.0 \mathrm{kcal} / \mathrm{mol})$, and Linoleoyl chloride $(-4.8 \mathrm{kcal} / \mathrm{mol})$. The detailed information was shown in Table 6. The MDT score of five ligands on PLA2G2A protein (PDB ID: 1KVO) was identified in the "Homo Sapiens" mode. It was exhibited that Lanosterol acetate $(-8.4 \mathrm{kcal} / \mathrm{mol})$ docked on PLA2G2A revealed the highest binding energy, followed by 1 -Palmitoylglycerol $(-6.8 \mathrm{kcal} / \mathrm{mol})$, Tricosanoic acid $(-5.9 \mathrm{kcal} / \mathrm{mol})$, Palmitic acid $(-5.4 \mathrm{kcal} / \mathrm{mol})$, and Linoleoyl chloride $(-4.8 \mathrm{kcal} / \mathrm{mol})$. The docking results were enlisted in Table 7. The MDT score of five ligands on PLA2G4A protein (PDB ID: 1BCI) was evaluated in the "Homo Sapiens" mode. It was revealed that 2-Linoleoylglycerol $(-4.9 \mathrm{kcal} / \mathrm{mol})$ showed the greatest binding energy, followed by cis-cis-cis-7, 10, 13 Hexadecatrienal $(-4.1 \mathrm{kcal} / \mathrm{mol})$, Linoleoyl chloride $(-4.0 \mathrm{kcal} / \mathrm{mol})$, Tricosanoic acid $(-3.6 \mathrm{kcal} / \mathrm{mol})$, and Palmitic acid (-3.3 kcal/mol). Interestingly, the MDT score of 5 compounds (D1-D5) on PLA2G4A demonstrated invalid affinity scores $\left(>-6.0 \mathrm{kcal}^{*} \mathrm{~mol}^{-1}\right)$ [42]; accordingly, we did not regard them as potential bioactives against gout. The docking detail information was presented in Table 8.

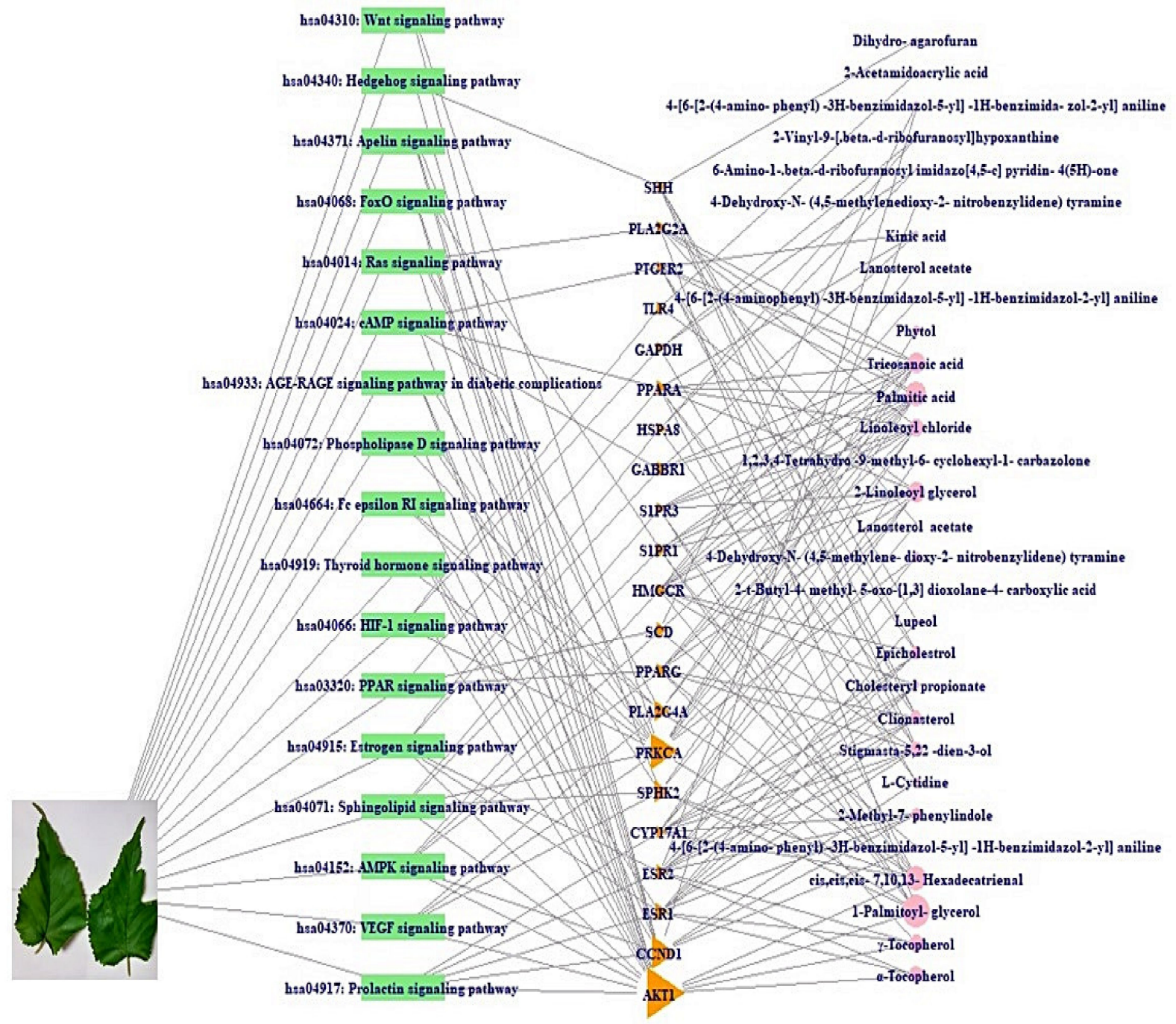

Figure 6. S-T-B networks of M. alba L. leaves. 
(A)

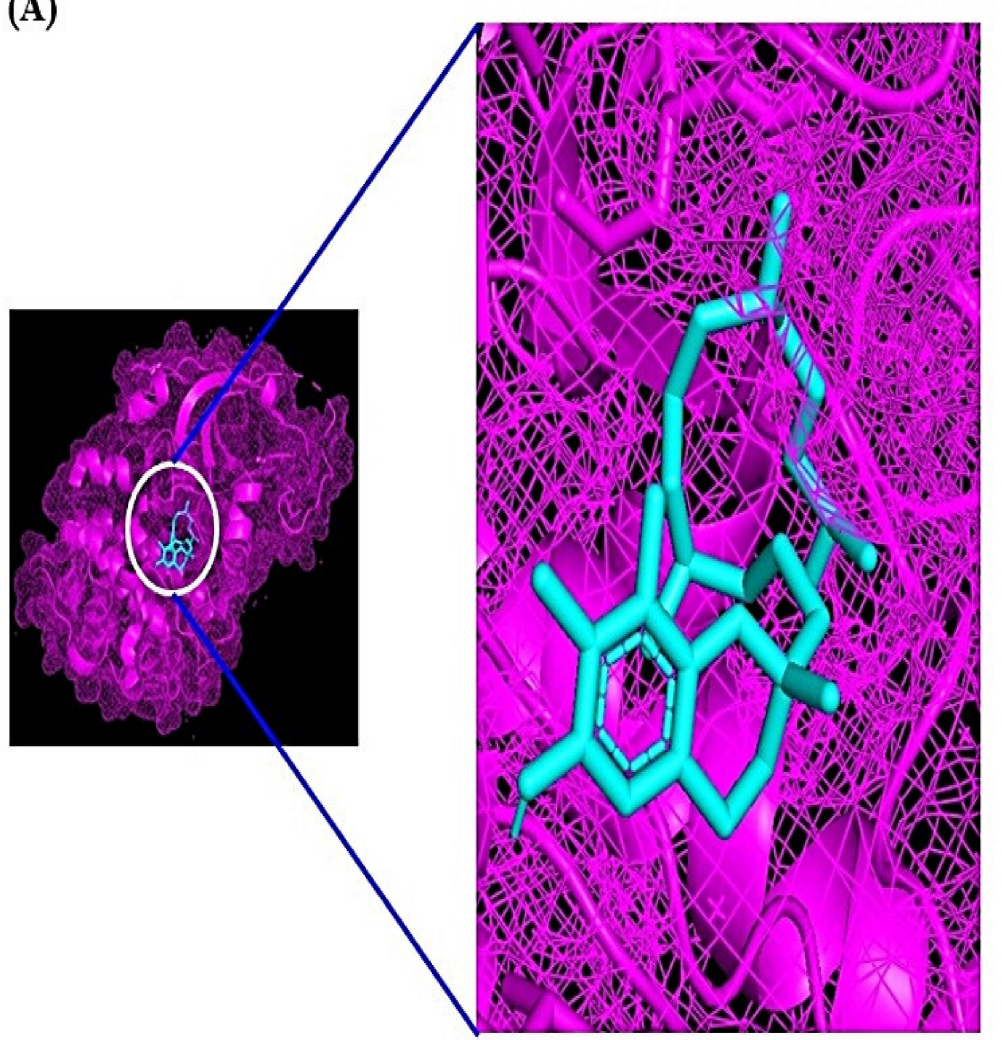

-AKTl(PDB ID: 4GVl): Magenta color

- $\gamma$-Tocopherol: Cyan color

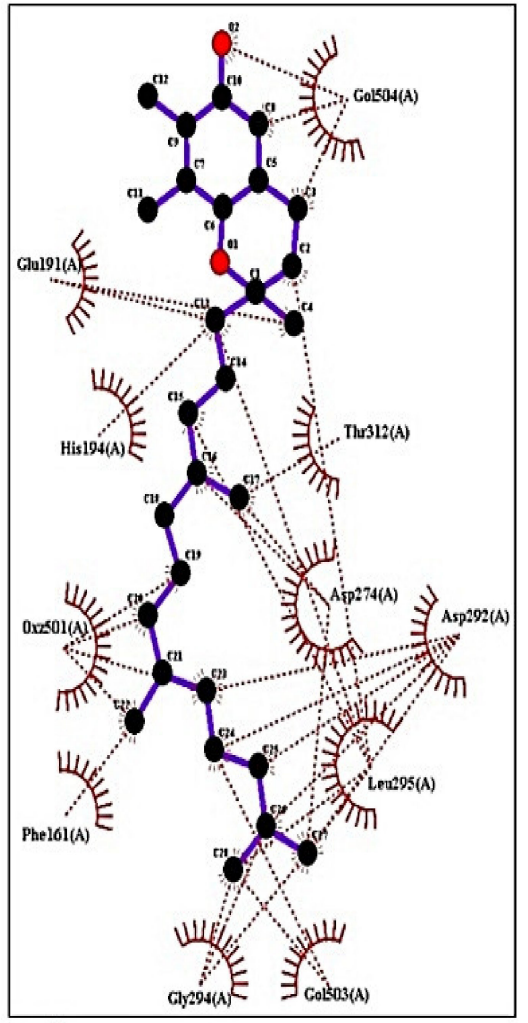

- : :Hydrophobic contacts

(B)

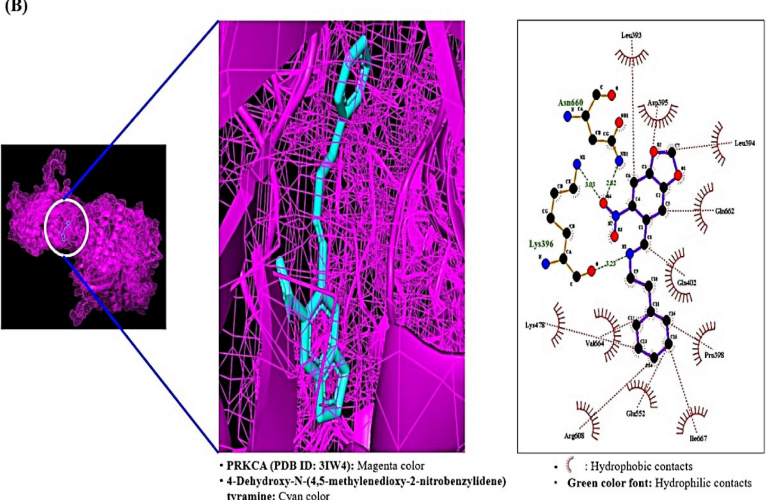

(C)
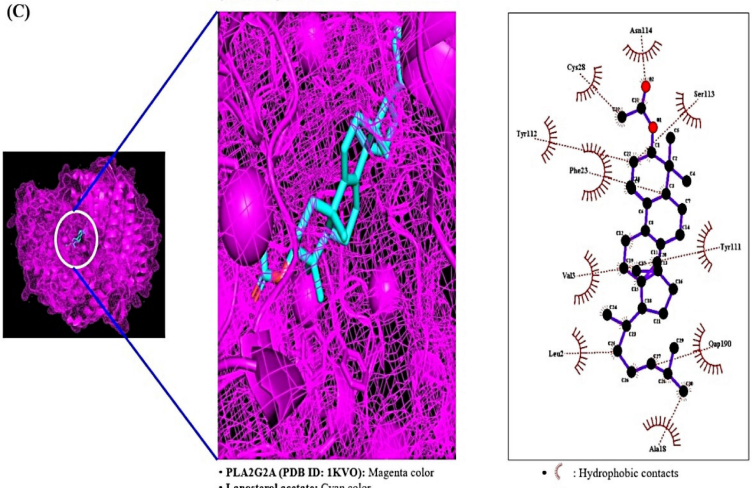

Figure 7. Molecular docking interaction between best docked compounds from SB and target proteins. (A) $\gamma$-Tocopherol on AKT1 (PDB ID: 4GV1). (B) 4-Dehydroxy-N-(4,5-methylenedioxy-2-nitrobenzylidene) tyramine on PRKCA (PDB ID: 3IW4). (C) Lanosterol acetate on PLA2G2A (PDB ID: 1KVO). 
Table 5. Binding energy and interactions of potential bioactives on AKT1 (PDB ID: 4GV1).

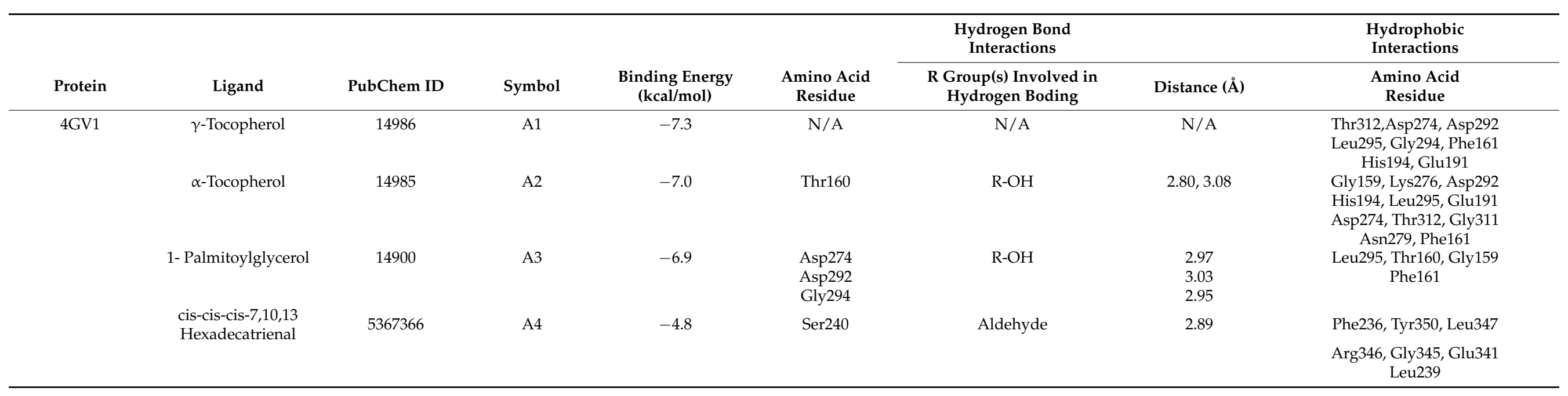

Table 6. Binding energy and interactions of potential bioactives on PRKCA (PDB ID: 3IW4).

\begin{tabular}{|c|c|c|c|c|c|c|c|c|}
\hline \multirow[b]{2}{*}{ Protein } & \multirow[b]{2}{*}{ Ligand } & \multirow[b]{2}{*}{ PubChem ID } & \multirow[b]{2}{*}{ Symbol } & \multirow[b]{2}{*}{$\begin{array}{l}\text { Binding Energy } \\
\text { (kcal/mol) }\end{array}$} & \multirow[b]{2}{*}{$\begin{array}{c}\text { Amino Acid } \\
\text { Residue }\end{array}$} & \multicolumn{2}{|l|}{$\begin{array}{l}\text { Hydrogen Bond } \\
\text { Interactions }\end{array}$} & $\begin{array}{l}\text { Hydrophobic } \\
\text { Interactions }\end{array}$ \\
\hline & & & & & & $\begin{array}{l}\text { R Group(s) Involved in } \\
\text { Hydrogen Boding }\end{array}$ & Distance (Å) & $\begin{array}{l}\text { Amino Acid } \\
\text { Residue }\end{array}$ \\
\hline \multirow[t]{14}{*}{ 3IW4 } & 1-Palmitoylglycerol & 14900 & B1 & -6.6 & ASP-395 & $\mathrm{R}-\mathrm{OH}$ & 2.88 & Val-664,Ile667,Pro666 \\
\hline & & & & & Leu-393 & $\mathrm{R}-\mathrm{OH}$ & 3.02 & Pro398.Gln402 \\
\hline & & & & & Lys-396 & R-OH, Aldehyde & $2.87,3.26$ & \\
\hline & & & & & Asn-660 & $\mathrm{R}-\mathrm{OH}$ & 3.06 & \\
\hline & 2-Linoleoylglycerol & 5365676 & B2 & -6.9 & Leu393 & $\mathrm{R}-\mathrm{OH}$ & 3.04 & Val-664, Pro666,Ile667 \\
\hline & & & & & Asp395 & $\mathrm{R}-\mathrm{OH}$ & 3.14 & Gln402,Pro398 \\
\hline & & & & & Gln662 & $\mathrm{R}-\mathrm{OH}$ & 3.06 & \\
\hline & & & & & Asn660 & R-OH, Carbonyl & $2.81,3.24$ & \\
\hline & Linoleoyl chloride & 9817754 & B3 & -4.8 & Lys396 & Haloform & 3.07 & Gln402, Pro398,Gln662 \\
\hline & Palmitic acid & 985 & B4 & -50 & & & & $\begin{array}{c}\text { Val664 } \\
\text { Val664 Gln662 His553 }\end{array}$ \\
\hline & Palmitic acia & 980 & B4 & -5.0 & $\begin{array}{l}\text { Lys396 } \\
\text { Asp395 }\end{array}$ & $\begin{array}{c}\text { Carbony, } \mathrm{K}-\mathrm{UH} \\
\mathrm{R}-\mathrm{OH}\end{array}$ & $\begin{array}{c}2.99,3.11 \\
3.10\end{array}$ & $\begin{array}{l}\text { Val664, Gln662, His553 } \\
\text { Ser549, Glu552, Gln402 }\end{array}$ \\
\hline & & & & & Leu393 & $\mathrm{R}-\mathrm{OH}$ & 3.15 & Pro398 \\
\hline & Tricosanoic acid & 17085 & B5 & -6.5 & Lys396 & Carbonyl, R-OH & $3.20,3.33$ & Gln402, Val664, Pro666 \\
\hline & & & & & Leu393 & R-OH & 2.89 & Pro398 \\
\hline
\end{tabular}


Table 6. Cont

\begin{tabular}{|c|c|c|c|c|c|c|c|c|}
\hline \multirow[b]{2}{*}{ Protein } & \multirow[b]{2}{*}{ Ligand } & \multirow[b]{2}{*}{ PubChem ID } & \multirow[b]{2}{*}{ Symbol } & \multirow[b]{2}{*}{$\begin{array}{l}\text { Binding Energy } \\
(\text { kcal } / \mathrm{mol})\end{array}$} & \multirow[b]{2}{*}{$\begin{array}{l}\text { Amino Acid } \\
\text { Residue }\end{array}$} & \multicolumn{2}{|l|}{$\begin{array}{c}\text { Hydrogen Bond } \\
\text { Interactions }\end{array}$} & $\begin{array}{l}\text { Hydrophobic } \\
\text { Interactions }\end{array}$ \\
\hline & & & & & & $\begin{array}{l}R \text { Group(s) Involved in } \\
\text { Hydrogen Boding }\end{array}$ & Distance ( $(\AA)$ & $\begin{array}{l}\text { Amino Acid } \\
\text { Residue }\end{array}$ \\
\hline & & & & & Leu393 & $\mathrm{R}-\mathrm{OH}$ & 3.00 & Glu552, Val664, Gln402 \\
\hline & & & & & Lys396 & $\mathrm{R}-\mathrm{OH}$ & 3.00 & \\
\hline & $\begin{array}{l}\text { 4-Dehydroxy-N-(4, } \\
\text { 5-methylenedioxy-2- } \\
\text { nitrobenzylidene) }\end{array}$ & 610062 & B7 & -8.4 & Lys-396 & Nitro, Imine & $3.03,3.23$ & Pro398, Ile667, Val664 \\
\hline & & & & & Asn-660 & Nitro & 2.82 & Glu552, Gln402, Gln662 \\
\hline
\end{tabular}

Table 7. Binding energy and interactions of potential bioactives on PLA2G2A (PDB ID: 1KVO).

\begin{tabular}{|c|c|c|c|c|c|c|c|c|}
\hline \multirow[b]{2}{*}{ Protein } & \multirow[b]{2}{*}{ Ligand } & \multirow[b]{2}{*}{ PubChem ID } & \multirow[b]{2}{*}{ Symbol } & \multirow[b]{2}{*}{$\begin{array}{l}\text { Binding Energy } \\
(\mathrm{kcal} / \mathrm{mol})\end{array}$} & \multirow[b]{2}{*}{$\begin{array}{l}\text { Amino Acid } \\
\text { Residue }\end{array}$} & \multicolumn{2}{|l|}{$\begin{array}{l}\text { Hydrogen Bond } \\
\text { Interactions }\end{array}$} & $\begin{array}{l}\text { Hydrophobic } \\
\text { Interactions }\end{array}$ \\
\hline & & & & & & $\begin{array}{l}\text { R Group(s) Involved in } \\
\text { Hydrogen Boding }\end{array}$ & Distance ( $(\AA)$ & Amino Acid Residue \\
\hline \multirow[t]{14}{*}{$1 \mathrm{KVO}$} & 1-Palmitoylglycerol & 14900 & $\mathrm{C} 1$ & -6.8 & Tyr112 & $\mathrm{R}-\mathrm{OH}$ & 2.06 & Val3, His6, Tyr111 \\
\hline & & & & & Gly25 & $\mathrm{R}-\mathrm{OH}$ & 2.32 & Ser113, Cys28, Gly22 \\
\hline & & & & & Phe23 & Ether & 2.33 & \\
\hline & & & & & Val30 & Ester & 2.98 & \\
\hline & & & & & Asn114 & $\mathrm{R}-\mathrm{OH}$ & $2.40,3.23$ & \\
\hline & Linoleoyl chloride & 9817754 & $\mathrm{C} 2$ & -4.8 & $\mathrm{~N} / \mathrm{A}$ & & $\mathrm{N} / \mathrm{A}$ & $\begin{array}{l}\text { Tyr111, Phe23,His6 } \\
\text { Leu2, Phe63, Val3 }\end{array}$ \\
\hline & Palmitic acid & 985 & $\mathrm{C} 3$ & -5.4 & Cys59 & $\mathrm{R}-\mathrm{OH}$ & 3.18 & Gly60, Phe-63, Lys62 \\
\hline & & & & & Thr61 & $\mathrm{R}-\mathrm{OH}$ & 2.96 & Glu55, Asn1, Phe63 \\
\hline & Tricosanoic acid & 17085 & $\mathrm{C} 4$ & -5.9 & Asn114 & $\mathrm{R}-\mathrm{OH}$ & 3.04 & Leu19, Glu16, Tyr111 \\
\hline & & & & & Cys28 & $\mathrm{R}-\mathrm{OH}$ & 2.99 & \\
\hline & & & & & Phe23 & $\mathrm{R}-\mathrm{OH}$ & 3.15 & \\
\hline & & & & & Gly25 & $\mathrm{R}-\mathrm{OH}$ & 2.29 & \\
\hline & & & & & Tyr112 & $\mathrm{R}-\mathrm{OH}$ & 2.06 & \\
\hline & Lanosterol acetate & 3036237 & C5 & -8.4 & $\mathrm{~N} / \mathrm{A}$ & $\mathrm{N} / \mathrm{A}$ & N/A & $\begin{array}{c}\text { Asn-114, Ser-113, Phe23 } \\
\text { Tyr-111, Leu2, Ala18 } \\
\text { Val3 }\end{array}$ \\
\hline
\end{tabular}


Table 8. Binding energy of potential bioactives on PLA2G4A (PDB ID: 1BCI).

\begin{tabular}{|c|c|c|c|c|c|c|c|c|}
\hline \multirow[b]{2}{*}{ Protein } & \multirow[b]{2}{*}{ Ligand } & \multirow[b]{2}{*}{ PubChem ID } & \multirow[b]{2}{*}{ Symbol } & \multirow[b]{2}{*}{$\begin{array}{l}\text { Binding Energy } \\
\text { (kcal/mol) }\end{array}$} & \multirow[b]{2}{*}{$\begin{array}{c}\text { Amino Acid } \\
\text { Residue }\end{array}$} & \multicolumn{2}{|l|}{$\begin{array}{l}\text { Hydrogen Bond } \\
\text { Interactions }\end{array}$} & Hydrophobic Interactions \\
\hline & & & & & & $\begin{array}{l}R \text { Group(s) Involved in } \\
\text { Hydrogen Boding }\end{array}$ & Distance (Å) & Amino Acid Residue \\
\hline \multirow[t]{7}{*}{$1 \mathrm{BCI}$} & 2-Linoleoylglycerol & 5365676 & D1 & -4.9 & $\mathrm{G} \ln 83$ & $\mathrm{R}-\mathrm{OH}$ & 3.22 & Tyr16, Pro54, Thr53 \\
\hline & & & & & Thr52 & $\mathrm{R}-\mathrm{OH}$ & 2.90 & Leu79 \\
\hline & & & & & Asp80 & R-OH & $2.87,3.19$ & \\
\hline & Linoleoyl chloride & 9817754 & D2 & -4.0 & Lys58 & Haloform & 3.04 & $\begin{array}{c}\text { Pro54, Ile78, Phe77 } \\
\text { Tyr16, Thr53 }\end{array}$ \\
\hline & Palmitic acid & 985 & D3 & -3.3 & His-62 & $\mathrm{R}-\mathrm{OH}$ & 3.14 & Ala94, Tyr45, Phe63 \\
\hline & $\begin{array}{l}\text { cis-cis-cis-7,10,13 } \\
\text { Hexadecatrienal }\end{array}$ & 5367366 & D5 & -4.1 & N/A & $\mathrm{N} / \mathrm{A}$ & $\mathrm{N} / \mathrm{A}$ & Asn95, Tyr96, Met98 \\
\hline & & & & & & & & $\begin{array}{c}\text { Glu100, Phe35, Val97 } \\
\text { Gly36 }\end{array}$ \\
\hline
\end{tabular}




\subsection{Linearity of Standard $\gamma$-Tocopherol}

Linearity was evaluated by the standard curve, determined by 4 different concentrations of $\gamma$-Tocopherol dissolved in $\mathrm{MeOH}$. The peak area was obtained to calculate the correlation coefficient of square linear regression analysis. The linearity of peak area responses versus concentrations was identified in the range of $4.048 \mathrm{mg} \mathrm{mL}^{-1}$ to $30.775 \mathrm{mg} \mathrm{mL}^{-1}$ $(r=0.99859, n=4)$ (Figure 8).
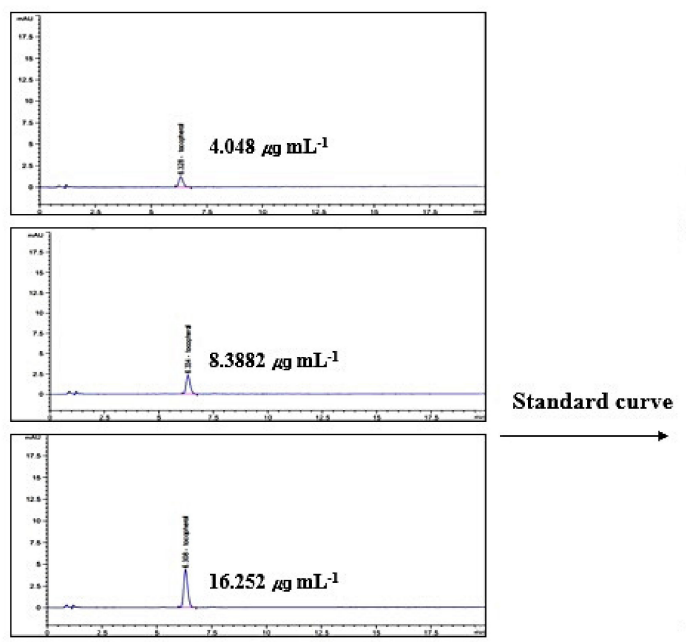

Standard curve
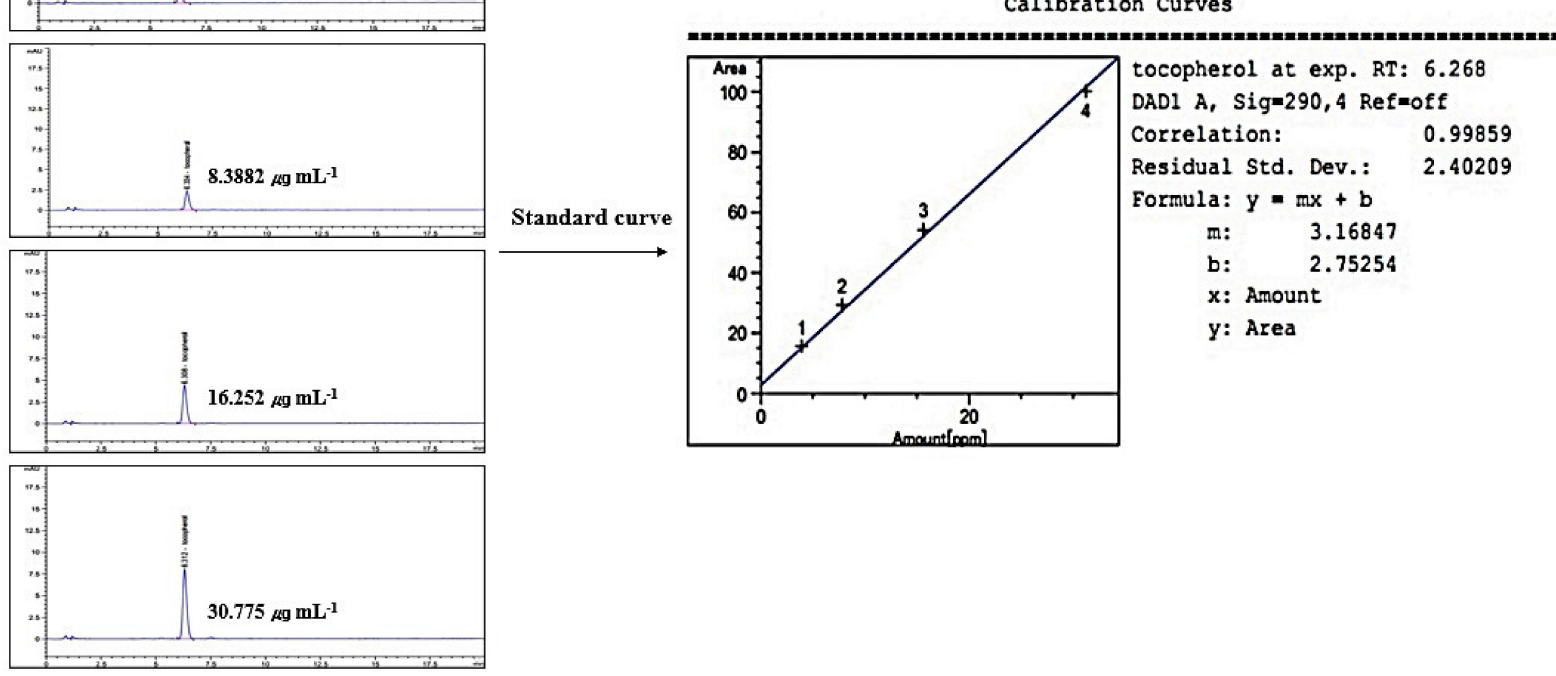

Figure 8. Standard curve for HPLC/UV analysis of $\gamma$-Tocopherol (wavelength: $290 \mathrm{~nm}$ ).

\subsection{The Identification of $\gamma$-Tocopherol from M. alba L. Leaves}

The retention time of $\gamma$-Tocopherol was $6.271 \mathrm{~min}$ in the HPLC analysis system, which overlapped exactly with the standard solution. The $\gamma$-Tocopherol amount was $9.077 \mathrm{mg} \mathrm{mL}^{-1}$ in M. alba L. leaves $\mathrm{MeOH}$ extraction $\left(20 \mathrm{mg} \mathrm{mL}^{-1}\right)$ (Figure 9). The ratio of $\gamma$-Tocopherol was comprised around $0.045 \%$ in HCLLs MeOH extract.

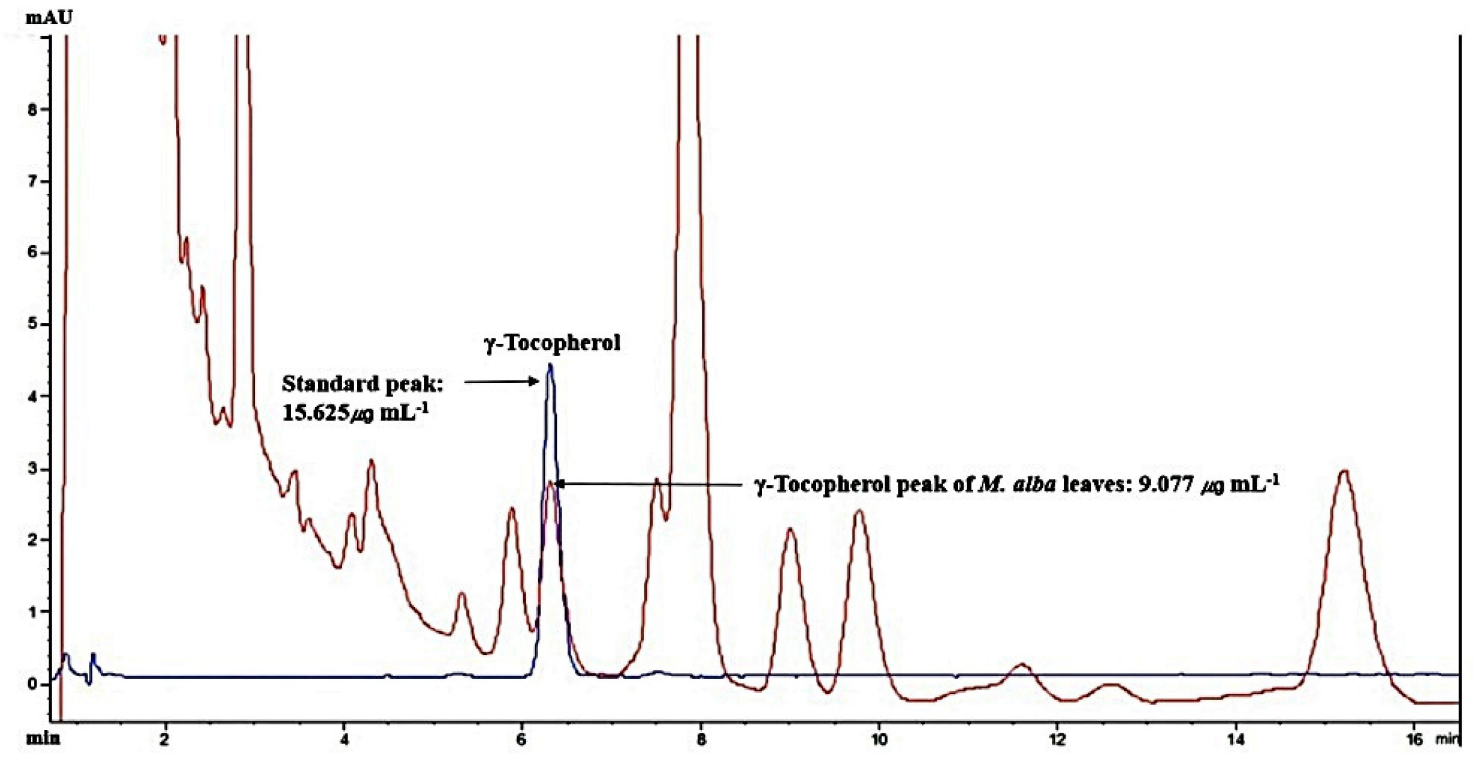

Figure 9. Overlapping HPLC chromatograms obtained by standard $\gamma$-Tocopherol (blue curve) and $\gamma$-Tocopherol (red curve) in M. alba L. leaves MeOH extraction, wavelength: $290 \mathrm{~nm}$. 


\subsection{Toxicological Properties of Selected Key Compounds}

Additionally, toxicological properties of the key three compounds ( $\gamma$-Tocopherol, 4Dehydroxy-N-(4,5-methylenedioxy-2-nitrobenzylidene) tyramine, and Lanosterol acetate) were predicted by admetSAR online tool. Our result indicated that the three compounds did not reveal Ames toxicity, carcinogenic properties, acute oral toxicity, and rat acute toxicity properties (Table 9 ).

Table 9. Toxicological properties of the key bioactives on AKT1 (PDB ID: 4GV1) in the molecular docking study.

\begin{tabular}{cccc}
\hline & \multicolumn{3}{c}{ Compound Name } \\
\cline { 2 - 4 } Parameters & $\gamma$-Tocopherol & $\begin{array}{c}\text { 4-Dehydroxy-N-(4, 5-methylenedioxy-2 } \\
\text {-nitrobenzylidene) tyramine }\end{array}$ & $\begin{array}{c}\text { Lanosterol } \\
\text { Acetate }\end{array}$ \\
\hline Ames toxicity & NAT & AT & NAT \\
Carcinogens & NC & NC & NC \\
Acute oral toxicity & III & III & III \\
Rat acute toxicity & 2.1598 & 2.6672 & 2.0477 \\
\hline
\end{tabular}

AT: Ames toxic; NAT: Non Ames toxic; NC: Non-carcinogenic; Category-II means ( $50 \mathrm{mg} / \mathrm{kg}>\mathrm{LD} 50<500 \mathrm{mg} / \mathrm{kg}$ ); Category-III means $(500 \mathrm{mg} / \mathrm{kg}>\mathrm{LD} 50<5000 \mathrm{mg} / \mathrm{kg})$.

\section{Discussion}

AKT1 is the highest degree (31) in PPI and the greatest degree (14) among 21 target proteins associated with 17 signaling pathways. Based on each target's degree value, AKT1 was regarded as the hub target of $M$. alba L. leaves against gout. A report demonstrated that AKT1-knockout-mice exposed noticeably reduced edema comparable in control groups; the inhibition of inflammation was related to a significant reduction in neutrophil and monocyte [43]. Among 26 compounds in M. alba L. leaves, $\gamma$-Tocopherol with the strongest affinity on AKT1 was the uppermost bioactive against gout. Vitamin $E$ reported in nature consists of four alpha $(\alpha)$, beta $(\beta)$, gamma $(\gamma)$, and delta $(\delta)$ - Tocopherol, both $\alpha$-Tocopherol and $\gamma$-Tocopherol have anti-inflammatory efficacy in vitro and in vivo, substances with $\gamma$-Tocopherol have stronger potency than $\alpha$-Tocopherol alone [44-47]. Among 17 signaling pathways, RAS signaling pathway was a hub signaling pathway based on rich factor with the lowest value on STRING analysis. The RAS signaling pathway can regulate IL-6 secretion; specifically, IL-6 production is associated with inflammation, immunity, and bone metabolism [48]. Network pharmacology analysis expounded that 17 signal pathways of M. alba L. leaves against gout were related to 26 compounds out of 36 compounds detected by GC-MS, including six prenol lipids ( $\alpha$-Tocopherol, $\gamma$-Tocopherol, Lupeol, Lanosterol acetate, Phytol, and Dihydroagarofuran). The ratio of prenol lipids to 26 compounds was close to $25 \%$, suggesting that prenol lipids were more significant than any other kind of compound for the amelioration of $M$. alba L. leaves on gout. It was reported that prenol lipids are involved in cell proliferation and differentiation in smooth muscle cell [49]. Other studies suggested that prenol lipids are the important regulator for inflammation and bone health [50-52].

The PPI displayed that 17 signaling pathways were directly associated with gout occurrence and development, implying that the 17 signaling pathways might be the molecular mechanisms of M. alba L. leaves against gout. Thus, the 17 signaling pathways connected to gout were briefly discussed as follows. PPAR (Peroxisome Proliferator-Activated Receptor) signaling pathway: PPAR- $\gamma$ (Peroxisome Proliferator-Activated Receptor-Gamma) expression on monocytes aggravated gouty arthritis and accelerated cytokine secretion [53]. RAS (Renin-Angiotensin System) signaling pathway: Uric acid is a leading causative element of gout, inducing oxidative stress via RAS activation [54]. It is evident that the inactivation of RAS may diminish the inflammatory level of gout. cAMP (cyclic Adenosine MonoPhosphate) signaling pathway: The increased cAMP level debilitated the MSU (Mono Sodium Urate)-induced activation of the Nod-like receptor protein 3 (NLRP3) signaling pathway, indicating the vital role of cAMP in the regulation of $\mathrm{P}_{2} \mathrm{Y}_{14}$ receptor $\left(\mathrm{P} 2 \mathrm{Y}_{14} \mathrm{R}\right)$ - 
mediated gouty arthritis [55]. HIF-1 (Hypoxia Inducible Factor-1) signaling pathway: MSU crystals increased the gene expression level of Hypoxia Inducible Factor $-1 \alpha(\mathrm{HIF}-1 \alpha)$ in Fibroblast-Like Synoviocytes (FLS), and its expression in FLS might be an indication of inflammation [56]. FoxO (Forkhead box O) signaling pathway: FoxO is a transcription factor to modulate AKT for IL-RA (Interleukin Receptor Antagonist) inhibition, which is an upstream controller to secrete cytokines [57]. Sphingolipid signaling pathway: Sphingolipids can ameliorate synovial inflammation and restore injured joints' responses [58]. Phospholipase D signaling pathway: Microcrystals-induced arthritis triggers phospholipase $\mathrm{D}$ in human neutrophils, and its activation was partially intolerance to colchicine used as gout treatment [59]. AMPK (AMP-activated Protein Kinase) signaling pathway: The consistent AMPK activation could diminish lysosomal NKA ( $\mathrm{Na}^{+}-\mathrm{K}^{+}$-ATPase) breakdown and sustain NKA function, thus relieving NKA inflammation and preserving tubular cells from high Uric acid-induced renal tubular damage [60]. Wnt (Wingless-INT) signaling pathway: Wnt signaling molecules and in vivo and in vitro animal studies suggest that Wnt signaling is an important therapeutic target for osteoarthritis, and the target tissues of Wnt signaling may be articular cartilage, synovium, and subchondral bone [61]. Hedgehog signaling pathway: The aberration of Hedgehog signaling regulation results in multiple bone diseases like heteroplasis, and thus, Hedgehog might be a promising biomarker for abnormal bone cartilage development [62]. VEGF (Vascular Endothelial Growth Factor) signaling pathway: A report suggested that VEGF counteracted properly pain responses and/or enhanced cartilage degeneration, synovitis, and osteophyte formation. Moreover, inhibition of VEGF signaling results in reduced pain [63]. Apelin (APLN) signaling pathway: APLN can control peripheral pain sensitivity sustained by APJ (APLN receptor) [64]. FceRI (Fc epsilon RI) signaling pathway: IgE (Immunoglobulin-E) mediated by FceRI signaling pathway inhibits bone remodelling due to mast cell activation, implicating gouty arthritis occurrence [65]. Estrogen signaling pathway: Estrogen treatment in rats has led to a dose-dependent cartilage weakness and a decrease in the extracellular matrix [66]. Prolactin signaling pathway: Prolactin treatment in rats diminished joint swelling, expanded trabecular bone area, reduced osteoclast density as well as protected bone loss in inflammatory arthritis [67]. Thyroid signaling pathway: Hyperthyroidism decreases the proinflammatory activities of monocytes and macrophages, which aggravate inflammation on gouty arthritis [68-70]. AGE-RAGE (Advanced Glycation End products- Receptor of Advanced Glycation End products) signaling pathway in diabetic complications: A study suggested that uric acid overexpressed the AGE-RAGE, which increased secretion of the inflammatory cytokine [71]. These signaling pathways imply interaction of multi-compound, multi-target, and multi-mechanism in the anti-gout activity of M. alba L. leaves.

Based on MDT, a hub bioactive of M. alba L. leaves against gout is $\gamma$-Tocopherol which had the strongest affinity on AKT1 (considered as a hub target against gout). The AKT1 of M. alba L. leaves against gout was directly connected to 14 out of 17 signaling pathways by the RAS signaling pathway, suggesting that the RAS signaling pathway might be a hub signaling pathway M. alba L. leaves against gout. A bone joint is the central disease region in gout patients, and its inflammatory arthritis is characterized by swelling, tenderness, and redness [72]. Moreover, gout patients indicated low anti-apoptotic target proteins (Bcl-2, Bcl- $\mathrm{X}_{\mathrm{L}}$ ) in synovial $\mathrm{T}$ cells, which is clear evidence of immunocompromised condition during gouty arthritis [73]. Recently, an animal experiment showed that colchicine (a common drug for gout) on macrophage in a mouse brain inhibits the RAS gene family with the inhibition of IL-1 $\beta$ (Interleukin 1 beta) [74]. The RAS inhibitors might promote anti-arthritis immunity in addition to targeting the macrophage cell's dependency on the RAS signaling [75]. It is clear evidence that inflammatory reaction around bone cartilage might be to control via RAS signaling pathway. A report concluded that $\gamma$-Tocopherol is vital in inhibiting inflammation-associated diseases like rheumatoid arthritis, asthma, and even hepatitis [44]. It is evident that $\gamma$-Tocopherol is bound to AKT1 (a hub target on RAS signaling pathway) to foster anti-gout arthritis by blocking the RAS signaling pathway. The PRKCA is related to chronic pain of human osteoarthritis and over-expressed mRNA 
abundance levels in an osteoarthritis rat model [76,77]. However, it is not reported that 4-Dehydroxy-N-(4, 5-methylenedioxy-2-nitrobenzylidene) tyramine on PRKCA functioned as an anti-inflammatory effect in immunology. The PLA2G2A over-represented in synovial fluid samples of gouty arthritis patients was identified via liquid chromatography tandem mass spectrometry (LC-MS/MS), compared to Ankylosing Spondylitis (AS) [78]. We suggest that lanosterol acetate on PLA2G2A might be a potent antagonist by blocking the RAS signaling pathway. The PLA2G4A plays an essential role in regulating inflammatory response with Cyclooxygenase-2 (COX-2) activation mirrored eicosanoid biosynthesis [79]. However, compounds of $M$. alba L. leaves related to PLA2G4A did not show attractive docking scores $(>-6.0 \mathrm{kcal} / \mathrm{mol})$. The cut-off of AutoDock Vina program was considered as active molecules (binding affinity value $<-6.0 \mathrm{kcal} / \mathrm{mol}$ ) [42]. Furthermore, according to the highest MD, three bioactives have been selected, specifically $\gamma$-Tocopherol, 4-Dehydroxy-N-(4,5-methylenedioxy-2-nitrobenzylidene) tyramine, and Lanosterol acetate, to clarify their physicochemical and toxicological properties. If any bioactives are not accepted by Lipinski's rule, it will not be evaluated as good oral bioavailability [80,81]. Our research showed that none of the bioactives, except "4-Dehydroxy-N-(4,5-methylenedioxy2-nitrobenzylidene) tyramine", violated Ames, which demonstrates good oral bioavailability. The study of toxicology suggested that none of the bioactives constitute a risk of Ames toxicity, carcinogenic properties, acute oral toxicity, and rat acute toxicity. To sum up, all three bioactives could be potential drug candidates with good oral bioavailability against gout. Therefore, the key mechanism of $M$. alba L. leaves against gout might be to suppress the inflammasomes in synovial fluids by inhibiting AKT1 by $\gamma$-Tocopherol, PRKCA by 4-Dehydroxy-N-(4,5-methylenedioxy-2-nitrobenzylidene) tyramine, and PLA2G2A by Lanosterol acetate on the RAS signaling pathway (Figure 10).

\section{RAS signaling pathway}

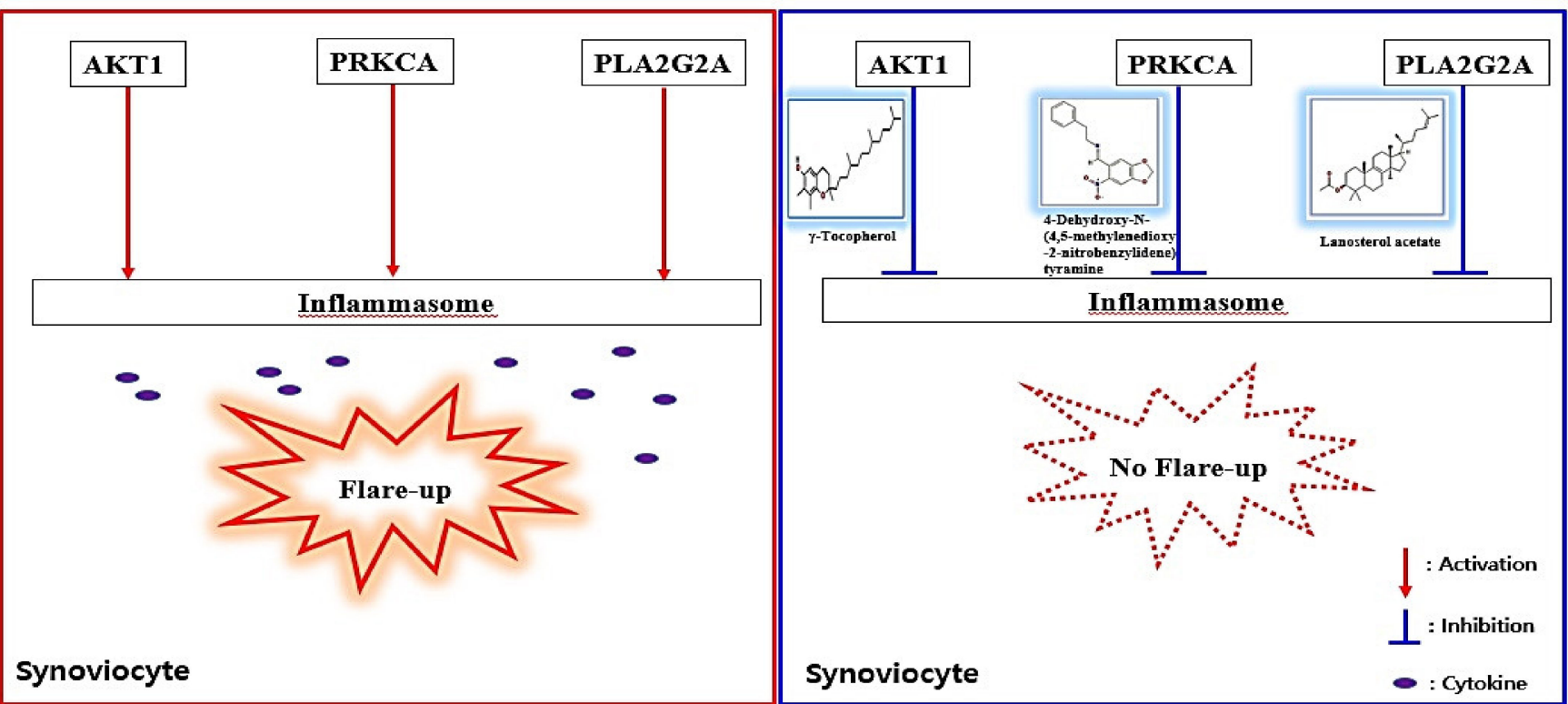

Figure 10. Summary figure of key findings in the study. 


\section{Materials and Methods}

\subsection{Plant Material Collection and Classification}

The M. alba L. leaves were collected from (Latitude: 36. 666700, Longitude: 128. 510729, Gyeongsangbuk-do, Republic of Korea, in August 2020, the plant was identified by Dr. Dong Ha Cho, Plant biologist and Professor, Department of Bio-Health Convergence, College of Biomedical Science, Kangwon National University. A voucher number (CRT 103) has been stored at Kenaf Corporation in the Department of Bio-Health Convergence, and the material can be used only for research purposes.

\subsection{Plant Preparation, Extraction}

The experimental M. alba L. leaves were harvested in May 2020 before fructifying. The growth stage of their leaves was fully grown at $8 \sim 12 \mathrm{~cm}$. The dried leaves $(20 \mathrm{~g})$ at room temperature $\left(20 \sim 22^{\circ} \mathrm{C}\right)$ for 7 days were soaked in $500 \mathrm{~mL}$ of methanol (Daejung, Siheung city, Korea). The extraction was carried out in a sealed bottle for 3 days and repeated 3 times at room temperature $\left(20 \sim 22^{\circ} \mathrm{C}\right)$. During extraction, the sample was shaken several times to increase the yield rate. The methanol was evaporated using a vacuum evaporator (IKA, Staufen city, Germany). The evaporated sample was dried under a hot water bath (IKA, Staufen city, Germany) at $40^{\circ} \mathrm{C}$.

\subsection{GC-MS Condition}

The analysis was carried out using the GC-MS system (Agilent 7890A, 5975C Agilent Technologies Inc., Santa Rosa, CA, USA) equipped with a DB-5 capillary column $(30 \mathrm{~m} \times 0.25 \mathrm{~mm} \times 0.25 \mu \mathrm{m})$. Firstly, the GC-MS instrument was maintained at a temperature of $100{ }^{\circ} \mathrm{C}$ for $2.1 \mathrm{~min}$. The temperature rose to $300{ }^{\circ} \mathrm{C}$ at the rate of $25^{\circ} \mathrm{C} / \mathrm{min}$ and was maintained for $10 \mathrm{~min}$ at the end of this period. Injection port temperature and helium flow rate were maintained as $250{ }^{\circ} \mathrm{C}$ and $1.5 \mathrm{~mL} / \mathrm{min}$. The samples injected in split mode at 10:1, and the ionization voltage was $70 \mathrm{eV}$. MS scan range was set at $35-550(\mathrm{~m} / \mathrm{z})$, and the fragmentation patterns of mass spectra compared in W8N05ST Library MS database. The relative peak area of each compound in the chromatogram was calculated on each compound percentage. ChemStation integrated algorithms were used as the concept of integration (analyzed 11 February 2021) [82].

\subsection{GC-MS Compounds in M. alba L. Leaves and Lipinski's Rule}

The species of chemical compounds from M. alba L. leaves were detected through GCMS. The compounds identified by GC-MS input into the PubChem (https:/ / pubchem.ncbi. nlm.nih.gov/) (accessed on 17 February 2021) to identify SMILES (Simplified Molecular Input Line Entry System). The identification of the "Drug-likeness" property is based on Lipinski's rule in SwissADME (http:/ / www.swissadme.ch/) (accessed on 20 February 2021) [83]. Moreover, the topological polar surface area (TPSA) value evaluates the ligands' cell permeability identified by SwissADME; generally, its permeability is typically limited when the TPSA value exceeds $140 \AA^{2}$ [84].

\subsection{Target Proteins Associated with Bioactives or Gout}

The bioactives accepted by Lipinski's rule input SMILE format into the two databases: SEA (Similarity Ensemble Approach) (http://sea.bkslab.org/) (accessed on 22 February 2021) [85] and STP (SwissTargetPrediction) (http:/ / www.swisstargetprediction.ch/) (accessed on 22 February 2021) [86] with "Homo Sapiens" setting. The target proteinscompounds interaction obtained by the two cheminformatics have been confirmed as powerful tools to be validated experimentally: SEA showed an accuracy rate of $80 \%$ out of novel drug candidates, and STP demonstrated that predictive target proteins of cudraflavone $\mathrm{C}$ was found via STP, thereby, validated by experiment $[87,88]$. Taken together, we assured that the novel new target(s) and mechanisms(s) against gout would be discovered by utilizing the validated data. Target proteins involved in gout were identified by two bioinformatics-DisGeNET (https:/ / www.disgenet.org/search) (accessed on 2 March 
2021) and OMIM (https: / / www.ncbi.nlm.nih.gov/omim) (accessed on 3 March 2021). The overlapping target proteins between drug-likeness compounds of $M$. alba L. leaves and gout-targeted proteins were identified and visualized on the Venn diagram by VENNY 2.1 (https:/ / bioinfogp.cnb.csic.es/tools/venny/) (accessed on 4 March 2021).

\subsection{Network Construction of Overlapping Target Proteins and Identification of Rich Factor}

Final overlapping target proteins were visualized through STRING (https:/ / string$\mathrm{db} .0 r g /$ ) analysis (accessed on 5 March 2021) [89]. The overlapping target proteins were closely co-expressed, and thus, signaling pathways associated with the overlapping target proteins were conceptualized by R Package bubble chart analysis. Based on rich factor and false discovery rate (FDR < 0.05), a hub signaling pathway of Morus alba (M. alba) L. leaves against gout were selected.

\subsection{A Signaling Pathway-Target Protein-Bioactive (S-T-B) Networks Construction}

The S-T-B networks were used to construct a size map, based on degree of values. In the network map, green rectangles (nodes) stood for signaling pathways; pink triangles (nodes) stood for target proteins, and orange circles (nodes) stood for bioactives; its circle size represented degree value. The size of pink triangles represented the number of connectivity with signaling pathways; the size of orange circles represented the number of connectivity with target proteins. The merged networks were constructed by using RPackage.

\subsection{Bioactives Preparation for MDT on a Hub Signaling Pathway}

The bioactives connected to a hub signaling pathway were converted sdf from PubChem into .pdb format using Pymol, finally, they were converted into .pdbqt format through Autodock.

\subsection{Target Proteins Preparation for $M D T$}

Four target proteins of gout i.e., AKT1 (PDB ID: 4GV1), PRKCA (PDB ID: 3IW4), PLA2G2A (PDB ID: 1KVO), PLA2G4A (PDB ID: 1BCI) were identified on STRING through RCSB PDB (https:/ / www.rcsb.org/) (accessed on 7 March 2021). The proteins selected as .PDB format was converted into .pdbqt format via Autodock (http:/ / autodock.scripps. edu/) (accessed on 7 March 2021).

\subsection{MDT of Bioactives on Target Proteins Associated with a Hub Signaling Pathway}

The ligand molecules were docked with target proteins utilizing autodock 4 by settingup 4 energy range and 8 exhaustiveness as default to obtain 10 different poses of ligand molecules [90]. The center of each target was AKT1 $(x=6.313, y=-7.926, z=17.198)$, PRKCA $(x=-14.059, y=38.224, z=32.319)$, PLA2G2A $(x=-48.436, y=71.878, z=47.001)$, PLA2G4A $(x=-0.058, y=0.077, z=0.285)$. The active site's grid box size was $x=40 \AA$, $\mathrm{y}=40 \AA, \mathrm{z}=40 \AA$. The 2D binding interactions was identified through LigPlot+ v.2.2 (https://www.ebi.ac.uk/thornton-srv/software/LigPlus/) (accessed on 9 March 2021). After docking, ligands with the lowest Gibbs free energy were selected to visualize the ligand-protein docking in Pymol.

\subsection{Chemicals and Reagents for HPLC Analysis}

Standard $\gamma$-Tocopherol was purchased from Sigma Aldrich (St. Louis, MO, USA). HPLC grade $\mathrm{MeOH}$ was obtained from Burdick \& Jackson. Ultrapure water obtained using a Milli-Q UF-Plus instrumentation (Millipore, MA, USA) was utilized to prepare all solutions for the method. 


\subsection{Instrumentation and Chromatographic Conditions}

HPLC Agilent 1260 series chromatographic instrumentation was used for this research. Data was collected and processed with Agilent 1260 chemstation. The HPLC system was equipped with an injection valve, quaternary gradient pump system, and UV dual $\lambda$ absorbance detector. Chromatographic separation was performed on a C18 column $4.6 \times 150 \mathrm{~mm}, 3.5 \mu \mathrm{m}$. The mobile phase was isocratic $\mathrm{MeOH} 98 \%(98: 2, v / v, \mathrm{MeOH}$ : water) at a flow rate of $2 \mathrm{~mL} \mathrm{~min}^{-1}$. Its analysis performed at ambient temperature, and detection was made at $290 \mathrm{~nm}$. The injected volume was $20 \mu \mathrm{L}$.

\subsection{Preparation of Standard Solution}

A stock solution of standard ( $\gamma$-Tocopherol) was prepared in $\mathrm{MeOH}$. The prepared stock solution concentration was made $3.906,7.813,15.626$, and $31.250 \mathrm{ppm}$ to plot the standard curve.

\subsection{Preparation of Plant Extraction for HPLC Analysis}

The $600 \mathrm{mg}$ of M. alba L. leaves $\mathrm{MeOH}$ extraction was taken in a flask, $30 \mathrm{~mL}$ of $\mathrm{MeOH}$ was added and kept for $3 \mathrm{~h}$. After shaking several times, the extraction was left for 5 days at room temperature. The solution of the flask was filtered through a Whatman No. 1 filter paper. The filtered solution was passed through a $0.2 \mu \mathrm{m}$ syringe filter and performed HPLC analysis.

\subsection{Toxicological Properties Prediction by admetSAR}

Toxicological properties of the key compounds were established using the admetSAR web-service tool (http:/ / lmmd.ecust.edu.cn/admetsar1/predict/) (accessed on 12 March 2021) because toxicity is a central element to develop new drugs. In the current study, Ames toxicity, carcinogenic properties, acute oral toxicity, and rat acute toxicity were predicted by admetSAR.

\section{Conclusions}

The bioactives and mechanism of M. alba L. leaves were firstly investigated through network pharmacology. The finding of this research suggested that $\gamma$-Tocopherol $(-7.3 \mathrm{kcal} / \mathrm{mol})$ on AKT1 (a hub target), 4-Dehydroxy-N-(4,5-methylenedioxy-2-nitrobenzylidene)tyramine $(-8.4 \mathrm{kcal} / \mathrm{mol})$ on PRKCA, and Lanosterol acetate $(-8.4 \mathrm{kcal} / \mathrm{mol})$ on PLA2G2A had the highest MDT, on each target. The five compounds associated with PLA2G4A did not manifest a valid MDT score. Thus, bioactives and target proteins of $M$. alba L. leaves against gout were connected to three target proteins. Hence, the three compounds, particularly $\gamma$-Tocopherol and AKT1, were regarded as the most significant bioactive and a hub target, respectively. Moreover, the promising mechanism of M. alba L. leaves against gout were connected to 17 signaling pathways, and a hub mechanism against gout might be to inhibit anti-arthritis immunity in synoviocytes by blocking the RAS signaling pathway. Overall, this research provides scientific evidence to support the therapeutic efficacy of $M$. alba L. leaves on gout and expounds new insights of bioactives, interactive target proteins, and mechanism(s) of M. alba L. leaves against gout.

Supplementary Materials: The following are available online at https://www.mdpi.com/article/ $10.3390 / \mathrm{ijms} 22179372 / \mathrm{s} 1$.

Author Contributions: Conceptualization, Methodology, Formal analysis, Investigation, Visualization, Data Curation, Writing—Original Draft, K.K.O.; Software, Investigation, Data Curation, K.K.O. and M.A.; Validation, Writing_-Review \& Editing, M.A.; Supervision, Project administration, D.H.C. All authors have read and agreed to the published version of the manuscript.

Funding: This research did not receive any specific grant from funding agencies in the public, commercial, or not-for-profit sectors.

Institutional Review Board Statement: Not applicable. 
Informed Consent Statement: Not applicable.

Data Availability Statement: All data generated or analyzed during this study are included in this published article (and its Supplementary Information files).

Acknowledgments: This research was acknowledged by the Department of Bio-Health Convergence, College of Biomedical Science, Kangwon National University, Chuncheon 24341, Republic of Korea.

Conflicts of Interest: There are no conflicts of interest declared.

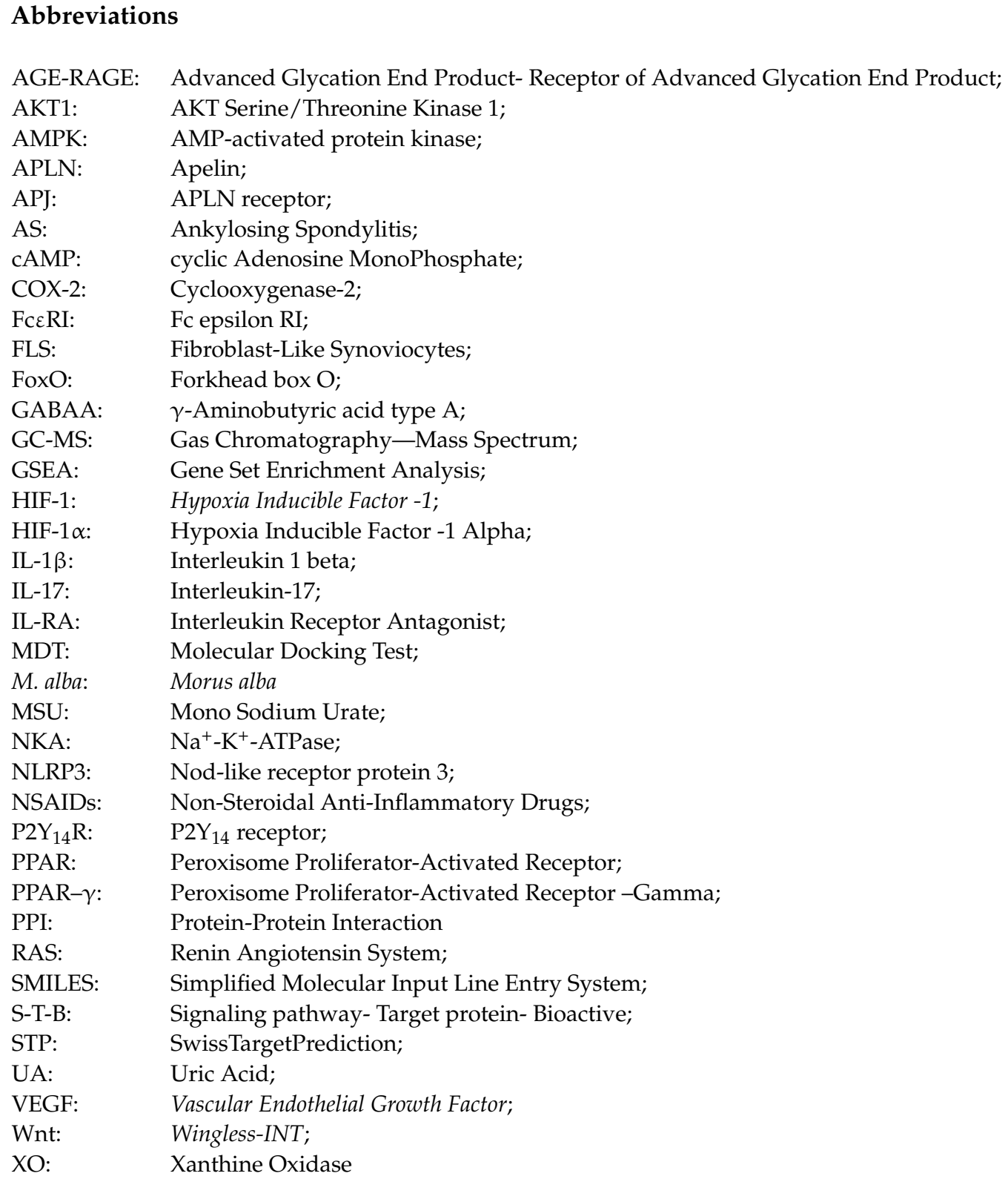

\section{References}

1. $\quad$ Dalbeth, N.; Choi, H.K.; Joosten, L.A.B.; Khanna, P.P.; Matsuo, H.; Perez-Ruiz, F.; Stamp, L.K. Gout. Nat. Rev. Dis. Primers 2019, 5, 1-17. [CrossRef]

2. Busso, N.; So, A. Mechanisms of inflammation in gout. Arthritis Res. Ther. 2010, 12, 206. [CrossRef] [PubMed]

3. Choi, H.K.; Niu, J.; Neogi, T.; Chen, C.A.; Chaisson, C.; Hunter, D.; Zhang, Y. Nocturnal risk of gout attacks. Arthritis Rheumatol. 2015, 67, 555-562. [CrossRef]

4. Towiwat, P.; Li, Z.-G. The association of vitamin C, alcohol, coffee, tea, milk and yogurt with uric acid and gout. Int. J. Rheum. Dis. 2015, 18, 495-501. [CrossRef] 
5. Kakutani-Hatayama, M.; Kadoya, M.; Okazaki, H.; Kurajoh, M.; Shoji, T.; Koyama, H.; Tsutsumi, Z.; Moriwaki, Y.; Namba, M.; Yamamoto, T. Nonpharmacological Management of Gout and Hyperuricemia: Hints for Better Lifestyle. Am. J. Lifestyle Med. 2017, 11, 321-329. [CrossRef] [PubMed]

6. Chi, X.; Zhang, H.; Zhang, S.; Ma, K. Chinese herbal medicine for gout: A review of the clinical evidence and pharmacological mechanisms. Chin. Med. 2020, 15, 17. [CrossRef]

7. Qaseem, A.; Harris, R.P.; Forciea, M.A.; Denberg, T.D.; Barry, M.J.; Boyd, C.; Chow, R.D.; Fitterman, N.; Humphrey, L.L.; Kansagara, D.; et al. Management of acute and recurrent gout: A clinical practice guideline from the American College of Physicians. Ann. Intern. Med. 2017, 166, 58-68. [CrossRef] [PubMed]

8. Cronstein, B.N.; Terkeltaub, R. The inflammatory process of gout and its treatment. Arthritis Res. Ther. 2006, 8, S3. [CrossRef] [PubMed]

9. Hussain, F.; Rana, Z.; Shafique, H.; Malik, A.; Hussain, Z. Phytopharmacological potential of different species of Morus alba and their bioactive phytochemicals: A review. Asian Pac. J. Trop. Biomed. 2017, 7, 950-956. [CrossRef]

10. Kadam, R.A.; Dhumal, N.D.; Khyade, V.B. The Mulberry, Morus alba (L.): The Medicinal Herbal Source for Human Health. Int. J. Curr. Microbiol. Appl. Sci. 2019, 8, 2941-2964. [CrossRef]

11. Kujawska, M.; Ewertowska, M.; Adamska, T.; Ignatowicz, E.; Flaczyk, E.; Przeor, M.; Kurpik, M.; Liebert, J.J. Protective effect of Morus alba leaf extract on N-nitrosodiethylamine-induced hepatocarcinogenesis in rats. In Vivo 2016, 30, 807-812. [CrossRef] [PubMed]

12. Wu, Y.X.; Kim, Y.J.; Kwon, T.H.; Tan, C.P.; Son, K.H.; Kim, T. Anti-inflammatory effects of mulberry (Morus alba L.) root bark and its active compounds. Nat. Prod. Res. 2020, 34, 1786-1790. [CrossRef] [PubMed]

13. Chan, E.W.C.; Lye, P.Y.; Wong, S.K. Phytochemistry, pharmacology, and clinical trials of Morus alba. Chin. J. Nat. Med. 2016, 14, 17-30.

14. Dkhil, M.A.; Bauomy, A.A.; Diab, M.S.M.; Al-Quraishy, S. The Antioxidant Effect of Morus alba Leaves Extract on Kidney, Testes, Spleen and Intestine of Mice. Pak. J. Zool. 2015, 47, 393-397.

15. Ling, X.; Bochu, W. A review of phytotherapy of gout: Perspective of new pharmacological treatments. Pharmazie 2014, 69, 243-256.

16. Wan, L.; Gang, C.; Jian, S.; Yin, X.J.; Zhu, H. Antioxidant and Xanthine Oxidase Inhibitory Properties and Lc-Ms/Ms Identification of Compounds of Ethanolic Extract from Mulberry Leaves. Acta Sci. Pol. Technol. Aliment. 2018, 17, 313-319. [CrossRef] [PubMed]

17. Li, H.; Yun, S.-B.; Shin, S.H.; Jeong, J.-M. Effect of Mulberry Extract Complex on Degenerative Arthritis in Vivo Models. J. Korean Soc. Food Sci. Nutr. 2016, 45, 634-641. [CrossRef]

18. Yimam, M.; Lee, Y.-C.; Moore, B.; Jiao, P.; Hong, M.; Nam, J.-B.; Kim, M.-R.; Hyun, E.-J.; Chu, M.; Brownell, L. Analgesic and anti-inflammatory effects of UP1304, a botanical composite containing standardized extracts of Curcuma longa and Morus alba. J. Integr. Med. 2016, 14, 60-68. [CrossRef]

19. Eo, H.J.; Park, J.H.; Park, G.H.; Lee, M.H.; Lee, J.R.; Koo, J.S.; Jeong, J.B. Anti-inflammatory and anti-cancer activity of mulberry (Morus alba L.) root bark. BMC Complement. Altern. Med. 2014, 14, 200. [CrossRef]

20. Zhang, G.B.; Li, Q.Y.; Chen, Q.L.; Su, S.B. Network pharmacology: A new approach for Chinese herbal medicine research. Evid.-Based Complement. Altern. Med. 2013, 2013, 621423. [CrossRef]

21. Gomez-Verjan, J.C.; Ramírez-Aldana, R.; Pérez-Zepeda, M.U.; Quiroz-Baez, R.; Luna-López, A.; Gutierrez Robledo, L.M. Systems biology and network pharmacology of frailty reveal novel epigenetic targets and mechanisms. Sci. Rep. 2019, 17, 6-8. [CrossRef]

22. Li, W.; Yuan, G.; Pan, Y.; Wang, C.; Chen, H. Network pharmacology studies on the bioactive compounds and action mechanisms of natural products for the treatment of diabetes mellitus: A review. Front. Pharmacol. 2017, 8, 74. [CrossRef]

23. Zhang, R.; Zhu, X.; Bai, H.; Ning, K. Network pharmacology databases for traditional Chinese medicine: Review and assessment. Front. Pharmacol. 2019, 10, 123. [CrossRef] [PubMed]

24. Oh, K.; Adnan, M.; Cho, D. Uncovering Mechanisms of Zanthoxylum piperitum Fruits for the Alleviation of Rheumatoid Arthritis Based on Network Pharmacology. Biology 2021, 10, 703. [CrossRef]

25. Oh, K.K.; Adnan, M.; Ju, I.; Cho, D.H. A network pharmacology study on main chemical compounds from Hibiscus cannabinus L. leaves. RSC Adv. 2021, 11, 11062-11082. [CrossRef]

26. Oh, K.-K.; Adnan, M.; Cho, D.-H. Network Pharmacology Study to Interpret Signaling Pathways of Ilex cornuta Leaves against Obesity. Processes 2021, 9, 1106. [CrossRef]

27. PCIDB. Available online: https:/ / www.genome.jp/db/pcidb (accessed on 13 July 2021).

28. Yu, X.; Zhao, M.; Liu, F.; Zeng, S.; Hu, J. Identification of 2,3-dihydro-3,5-dihydroxy-6-methyl-4H-pyran-4-one as a strong antioxidant in glucose-histidine Maillard reaction products. Food Res. Int. 2013, 51, 397-403. [CrossRef]

29. Ramalakshmi, S.; Muthuchelian, K. Studies on cytotoxicity, phytotoxicity and volatile profile of flower extract of Tabebuia rosea (Bertol.) DC. Med. Plants 2012, 4, 154-161. [CrossRef]

30. Adnan, M.; Oh, K.K.; Azad, M.O.K.; Shin, M.H.; Wang, M.-H.; Cho, D.H. Kenaf (Hibiscus cannabinus L.) Leaves and Seed as a Potential Source of the Bioactive Compounds: Effects of Various Extraction Solvents on Biological Properties. Life 2020, 10, 223. [CrossRef]

31. Yang, F.; Oyeyinka, S.A.; Xu, W.; Ma, Y.; Zhou, S. In vitro bioaccessibility and physicochemical properties of phytosterol linoleic ester synthesized from soybean sterol and linoleic acid. LWT_Food Sci. Technol. 2018, 92, 265-271. [CrossRef] 
32. Sofi, M.S. Evaluation of Pro-Apoptotic Effects of $\beta$-Monolinolein on Metastatic Breast Cancer Cell Line MDA-MB-231. Asian J. Pharm. Clin. Res. 2019, 12, 235-240. [CrossRef]

33. Li, W.-R.; Shi, Q.-S.; Liang, Q.; Xie, X.-B.; Huang, X.-M.; Chen, Y.-B. Antibacterial Activity and Kinetics of Litsea cubeba Oil on Escherichia coli. PLoS ONE 2014, 9, e110983. [CrossRef]

34. Hadi, I.; Hussein, H.M. 18,19-Secoyohimban-19-oic acid. Int. J. Pharmacogn. Phytochem. Res. 2016, 8, 21-37.

35. Kiralan, S.S.; Dołu-Baykut, E.; Kittipongpittaya, K.; McClements, D.J.; Decker, E.A. Increased antioxidant efficacy of tocopherols by surfactant solubilization in oil-in-water emulsions. J. Agric. Food Chem. 2014, 62, 10561-10566. [CrossRef] [PubMed]

36. Kumar, S.B.; Krishna, S.; Pradeep, S.; Mathews, D.E.; Pattabiraman, R.; Murahari, M.; Murthy, T.P.K. Screening of natural compounds from Cyperus rotundus Linn against SARS-CoV-2 main protease (Mpro): An integrated computational approach. Comp. Biol. Med. 2021, 134, 104524. [CrossRef]

37. Cerqueira, F.; Watanadilok, R.; Sonchaeng, P.; Kijjoa, A.; Pinto, M.; Van Ufford, H.Q.; Kroes, B.; Beukelman, C.; Nascimento, M.S.J. Clionasterol: A potent inhibitor of complement component C1. Planta Med. 2003, 69, 174-176. [CrossRef] [PubMed]

38. Peng, W.; Li, D.; Zhang, M.; Ge, S.; Mo, B.; Li, S.; Ohkoshi, M. Characteristics of antibacterial molecular activities in poplar wood extractives. Saudi J. Biol. Sci. 2017, 24, 399-404. [CrossRef]

39. Wibowo, M.; Levrier, C.; Sadowski, M.C.; Nelson, C.C.; Wang, Q.; Holst, J.; Healy, P.C.; Hofmann, A.; Davis, R.A. Bioactive Dihydro- $\beta$-agarofuran Sesquiterpenoids from the Australian Rainforest Plant Maytenus bilocularis. J. Nat. Prod. 2016, 79, 1445-1453. [CrossRef]

40. Husein, H.A.; Alhasan, D.A.H.; Albadry, M.A.Z. In Vitro Antimicrobial Activity and GC-MS Analysis of Crude Aqueous Methanolic Extract Produced from Leaves of Eucalyptus species. Med. J. 2019, 17, 2019. [CrossRef]

41. Malinowska, M.; Miroslaw, B.; Sikora, E.; Ogonowski, J.; Wojtkiewicz, A.M.; Szaleniec, M.; Pasikowska-Piwko, M.; Eris, I. New lupeol esters as active substances in the treatment of skin damage. PLoS ONE 2019, 14, e0214216. [CrossRef]

42. Shityakov, S.; Förster, C. In silico predictive model to determine vector-mediated transport properties for the blood-brain barrier choline transporter. Adv. Appl. Bioinf. Chem. 2014, 7, 23-36. [CrossRef] [PubMed]

43. Di Lorenzo, A.; Fernández-Hernando, C.; Cirino, G.; Sessa, W.C. Akt1 is critical for acute inflammation and histamine-mediated vascular leakage. Proc. Natl. Acad. Sci. USA 2009, 106, 14552-14557. [CrossRef]

44. Reiter, E.; Jiang, Q.; Christen, S. Anti-inflammatory properties of $\alpha$ - and $\gamma$-tocopherol. Mol. Aspects Med. 2007, $28,668-691$. [CrossRef]

45. Kabir, M.I.; Adnan, M.; Rahman, M.M. Natural sources of tocotrienols: A note on absorption. J. Silico Vitr. Pharmacol 2017, 3, 1-5. [CrossRef]

46. Adnan, M.; Nazim Uddin Chy, M.; Mostafa Kamal, A.T.M.; Barlow, J.W.; Faruque, M.O.; Yang, X.; Uddin, S.B. Evaluation of antinociceptive and anti-inflammatory activities of the methanol extract of Holigarna caustica (Dennst.) Oken leaves. J. Ethnopharmacol. 2019, 236, 401-411. [CrossRef] [PubMed]

47. Chy, M.N.U.; Adnan, M.; Chowdhury, M.R.; Pagano, E.; Kamal, A.T.M.M.; Oh, K.K.; Cho, D.H.; Capasso, R. Central and peripheral pain intervention by Ophiorrhiza rugosa leaves: Potential underlying mechanisms and insight into the role of pain modulators. J. Ethnopharmacol. 2021, 276, 114182.

48. Ancrile, B.; Lim, K.H.; Counter, C.M. Oncogenic Ras-induced secretion of IL6 is required for tumorigenesis. Genes Dev. 2007, 21, 1714-1719. [CrossRef] [PubMed]

49. Ratnayake, W.N.; Galli, C. Fat and Fatty Acid Terminology, Methods of Analysis and Fat Digestion and Metabolism: A Background Review Paper. Ann. Nutr. Metab. 2009, 55, 8-43. [CrossRef]

50. Joshi, Y.B.; Praticò, D. Vitamin E in aging, dementia, and Alzheimer's disease. BioFactors 2012, 38, 90-97. [CrossRef] [PubMed]

51. Jiang, Q. Natural forms of vitamin E: Metabolism, antioxidant, and anti-inflammatory activities and their role in disease prevention and therapy. Free Radic. Biol. Med. 2014, 72, 76-90. [CrossRef]

52. Kidd, P.M. Vitamins D and K as Pleiotropic Nutrients: Clinical Importance to the Skeletal and Cardiovascular Systems and Preliminary Evidence for Synergy. Altern. Med. Rev. 2010, 15, 199-222.

53. Akahoshi, T.; Namai, R.; Murakami, Y.; Watanabe, M.; Matsui, T.; Nishimura, A.; Kitasato, H.; Kameya, T.; Kondo, H. Rapid induction of peroxisome proliferator-activated receptor $\gamma$ expression in human monocytes by monosodium urate monohydrate crystals. Arthritis Rheum. 2003, 48, 231-239. [CrossRef]

54. Wu, Q.; Chen, B.; Zhang, J.-X.; Zhang, Y.-P.; Wu, Q.-N. Uric acid induces oxidative stress via an activation of the renin-angiotensin system in 3T3-L1 adipocytes Solid-state nanopore DNA detection View project Uric acid induces oxidative stress via an activation of the renin-angiotensin system in 3T3-L1 adipocytes. Endocrine 2015, 48, 135-142. [CrossRef]

55. Li, H.; Jiang, W.; Ye, S.; Zhou, M.; Liu, C.; Yang, X.; Hao, K.; Hu, Q. P2Y14 receptor has a critical role in acute gouty arthritis by regulating pyroptosis of macrophages. Cell Death Dis. 2020, 11, 1-14. [CrossRef]

56. Zamudio-Cuevas, Y.; Fernández-Torres, J.; Martínez-Nava, G.A.; Martínez-Flores, K.; Ramírez Olvera, A.; Medina-Luna, D.; Hernández Pérez, A.D.; Landa-Solís, C.; López-Reyes, A. Highlight Article: Phagocytosis of monosodium urate crystals by human synoviocytes induces inflammation. Exp. Biol. Med. 2019, 244, 344-351. [CrossRef]

57. Crişan, T.O.; Cleophas, M.C.P.; Novakovic, B.; Erler, K.; Van De Veerdonk, F.L.; Stunnenberg, H.G.; Netea, M.G.; Dinarello, C.A.; Joosten, L.A.B. Uric acid priming in human monocytes is driven by the AKT-PRAS40 autophagy pathway. Proc. Natl. Acad. Sci. USA 2017, 114, 5485-5490. [CrossRef] [PubMed] 
58. Kosinska, M.K.; Liebisch, G.; Lochnit, G.; Wilhelm, J.; Klein, H.; Kaesser, U.; Lasczkowski, G.; Rickert, M.; Schmitz, G.; Steinmeyer, J. Sphingolipids in human synovial fluid-A lipidomic study. PLoS ONE 2014, 9, e91769. [CrossRef] [PubMed]

59. Naccache, P.H.; Bourgoin, S.; Plante, E.; Roberge, C.J.; De Medicis, R.; Lussier, A.; Poubelle, P.E. Crystal-induced neutrophil activation. II. evidence for the activation of a phosphatidylcholine-specific phospholipase D. Arthritis Rheum. 1993, 36, 117-125. [CrossRef]

60. Xiao, J.; Zhu, S.; Guan, H.; Zheng, Y.; Li, F.; Zhang, X.; Guo, H.; Wang, X.; Ye, Z. AMPK alleviates high uric acid-induced $\mathrm{Na}+\mathrm{K}+-\mathrm{ATPase}$ signaling impairment and cell injury in renal tubules. Exp. Mol. Med. 2019, 51, 1-14. [CrossRef]

61. Usami, Y.; Gunawardena, A.T.; Iwamoto, M.; Enomoto-Iwamoto, M. Wnt signaling in cartilage development and diseases: Lessons from animal studies. Lab. Investig. 2016, 96, 186-196. [CrossRef] [PubMed]

62. Yang, J.; Andre, P.; Ye, L.; Yang, Y.Z. The Hedgehog signalling pathway in bone formation. Int. J. Oral Sci. 2015, 7, 73-79. [CrossRef]

63. Llorián-Salvador, M.; González-Rodríguez, S. Painful understanding of VEGF. Front. Pharmacol. 2018, 9, 1267. [CrossRef]

64. Takano, S.; Uchida, K.; Inoue, G.; Matsumoto, T.; Aikawa, J.; Iwase, D.; Mukai, M.; Miyagi, M.; Takaso, M. Vascular endothelial growth factor expression and their action in the synovial membranes of patients with painful knee osteoarthritis. BMC Musculoskelet. Disord. 2018, 19, 204. [CrossRef] [PubMed]

65. Wang, Q.; Lepus, C.M.; Raghu, H.; Reber, L.L.; Tsai, M.M.; Wong, H.H.; Von Kaeppler, E.; Lingampalli, N.; Bloom, M.S.; Hu, N.; et al. Ige-mediated mast cell activation promotes inflammation and cartilage destruction in osteoarthritis. eLife 2019, 8, e39905. [CrossRef] [PubMed]

66. Kleine, S.A.; Budsberg, S.C. Synovial membrane receptors as therapeutic targets: A review of receptor localization, structure, and function. J. Orthop. Res. 2017, 35, 1589-1605. [CrossRef]

67. Ledesma-Colunga, M.G.; Adán, N.; Ortiz, G.; Solís-Gutiérrez, M.; López-Barrera, F.; de la Escalera, G.M.; Clapp, C. Prolactin blocks the expression of receptor activator of nuclear factor KB ligand and reduces osteoclastogenesis and bone loss in murine inflammatory arthritis. Arthritis Res. Ther. 2017, 19, 93. [CrossRef]

68. De Vito, P.; Incerpi, S.; Pedersen, J.Z.; Luly, P.; Davis, F.B.; Davis, P.J. Thyroid hormones as modulators of immune activities at the cellular level. Thyroid 2011, 21, 879-890. [CrossRef]

69. Jeong, J.H.; Hong, S.; Kwon, O.C.; Ghang, B.; Hwang, I.; Kim, Y.G.; Lee, C.K.; Yoo, B. CD14+ cells with the phenotype of infiltrated monocytes consist of distinct populations characterized by anti-inflammatory as well as pro-inflammatory activity in gouty arthritis. Front. Immunol. 2017, 8, 1260. [CrossRef]

70. Wang, J.; Yang, Q.; Zhang, Q.; Yin, C.; Zhou, L.; Zhou, J.; Wang, Y.; Mi, Q.S. Invariant natural killer T cells ameliorate monosodium urate crystal-induced gouty inflammation in mice. Front. Immunol. 2017, 8, 12. [CrossRef]

71. Cai, W.; Duan, X.M.; Liu, Y.; Yu, J.; Tang, Y.L.; Liu, Z.L.; Jiang, S.; Zhang, C.P.; Liu, J.Y.; Xu, J.X. Uric Acid Induces Endothelial Dysfunction by Activating the HMGB1/RAGE Signaling Pathway. Biomed Res. Int. 2017, 2017. [CrossRef] [PubMed]

72. Ragab, G.; Elshahaly, M.; Bardin, T. Gout: An old disease in new perspective-A review. J. Adv. Res. 2017, 8, 495-511. [CrossRef] [PubMed]

73. Salmon, M.; Scheel-Toellner, D.; Huissoon, A.P.; Pilling, D.; Shamsadeen, N.; Hyde, H.; D’Angeac, A.D.; Bacon, P.A.; Emery, P.; Akbar, A.N. Inhibition of T cell apoptosis in the rheumatoid synovium. J. Clin. Investig. 1997, 99, 439-446. [CrossRef] [PubMed]

74. Leung, Y.Y.; Yao Hui, L.L.; Kraus, V.B. Colchicine-Update on mechanisms of action and therapeutic uses. Semin. Arthritis Rheum. 2015, 45, 341-350. [CrossRef]

75. Reedquist, K.A. Signal Transduction Pathways in Chronic Inflammatory Autoimmune Disease: Small GTPases. Open Rheumatol. J. 2012, 6, 259-272. [CrossRef]

76. Warner, S.C.; Van Meurs, J.B.J.; Schiphof, D.; Bierma-Zeinstra, S.M.; Hofman, A.; Uitterlinden, A.G.; Richardson, H.; Jenkins, W.; Doherty, M.; Valdes, A.M. Genome-wide association scan of neuropathic pain symptoms post total joint replacement highlights a variant in the protein-kinase C gene. Eur. J. Hum. Genet. 2017, 25, 446-451. [CrossRef]

77. Korostynski, M.; Malek, N.; Piechota, M.; Starowicz, K. Cell-type-specific gene expression patterns in the knee cartilage in an osteoarthritis rat model. Funct. Integr. Genom. 2018, 18, 79-87. [CrossRef] [PubMed]

78. Lee, J.H.; Jung, J.H.; Kim, J.; Baek, W.K.; Rhee, J.; Kim, T.H.; Kim, S.H.; Kim, K.P.; Son, C.N.; Kim, J.S. Proteomic analysis of human synovial fluid reveals potential diagnostic biomarkers for ankylosing spondylitis. Clin. Proteom. 2020, 17, 20. [CrossRef]

79. Matsui, M.; Chu, Y.; Zhang, H.; Gagnon, K.T.; Shaikh, S.; Kuchimanchi, S.; Corey, D.R.; Janowski, B.A. Promoter RNA links transcriptional regulation of inflammatory pathway genes. Nucleic Acids Res. 2013, 41, 10086-10109. [CrossRef]

80. Lipinski, C.A.; Lombardo, F.; Dominy, B.W.; Feeney, P.J. Experimental and computational approaches to estimate solubility and permeability in drug discovery and development settings. Adv. Drug Deliv. Rev. 2001, 46, 3-26. [CrossRef]

81. Duffy, F.J.; Devocelle, M.; Shields, D.C. Computational approaches to developing short cyclic peptide modulators of proteinprotein interactions. In Computational Peptidology; Springer: Berlin/Heidelberg, Germany, 2015; pp. 241-271.

82. Oh, K.K.; Adnan, M.; Cho, D.H. Active ingredients and mechanisms of Phellinus linteus (grown on Rosa multiflora) for alleviation of Type 2 diabetes mellitus through network pharmacology. Gene 2020, 768, 145320. [CrossRef]

83. Daina, A.; Michielin, O.; Zoete, V. SwissADME: A free web tool to evaluate pharmacokinetics, drug-likeness and medicinal chemistry friendliness of small molecules. Sci. Rep. 2017, 7, srep42717. [CrossRef]

84. Matsson, P.; Kihlberg, J. How Big Is Too Big for Cell Permeability? J. Med. Chem. 2017, 60, 1662-1664. [CrossRef] [PubMed] 
85. Keiser, M.J.; Roth, B.L.; Armbruster, B.N.; Ernsberger, P.; Irwin, J.J.; Shoichet, B.K. Relating protein pharmacology by ligand chemistry. Nat. Biotechnol. 2007, 25, 197-206. [CrossRef] [PubMed]

86. Daina, A.; Michielin, O.; Zoete, V. SwissTargetPrediction: Updated data and new features for efficient prediction of protein targets of small molecules. Nucleic Acids Res. 2019, 47, W357-W3664. [CrossRef] [PubMed]

87. Singh, N.; Chaput, L.; Villoutreix, B.O. Virtual screening web servers: Designing chemical probes and drug candidates in the cyberspace. Brief. Bioinform. 2020, 22, 1790-1818. [CrossRef]

88. Soo, H.-C.; Chung, F.F.-L.; Lim, K.-H.; Yap, V.A.; Bradshaw, T.D.; Hii, L.-W.; Tan, S.-H.; See, S.-J.; Tan, Y.-F.; Leong, C.-O.; et al. Cudraflavone C Induces Tumor-Specific Apoptosis in Colorectal Cancer Cells through Inhibition of the Phosphoinositide 3-Kinase (PI3K)-AKT Pathway. PLoS ONE 2017, 12, e0170551. [CrossRef]

89. Szklarczyk, D.; Gable, A.L.; Lyon, D.; Junge, A.; Wyder, S.; Huerta-Cepas, J.; Simonovic, M.; Doncheva, N.T.; Morris, J.H.; Bork, P.; et al. STRING v11: Protein-protein association networks with increased coverage, supporting functional discovery in genome-wide experimental datasets. Nucleic Acids Res. 2019, 47, D607-D613. [CrossRef] [PubMed]

90. Khanal, P.; Patil, B.M.; Chand, J.; Naaz, Y. Anthraquinone Derivatives as an Immune Booster and their Therapeutic Option Against COVID-19. Nat. Prod. Bioprospect. 2020, 10, 325-335. [CrossRef] 